\title{
Refractory thrombocytopenia, recurrent abortions and inferior vena cava thrombosis
}

\author{
Atul Kakar, Ved Prakash
}

A 23-year-old woman was admitted in January 1983 with complaints of purpuric spots all over her body for 4 days prior to admission. She had been married for 3 years, had undergone three spontaneous abortions (one first trimester and two second trimester), and had one live birth. On examination, she had no splenomegaly. Investigations revealed a haemoglobin of $11.2 \mathrm{~g} / \mathrm{dl}$, total leukocyte count $4 \times 10^{9} / 1$ (polymorphs $64 \%$, lymphocytes $36 \%$ ), erythrocyte sedimentation rate $12 \mathrm{~mm}$ in first hour, platelets $40 \times 10^{9} / 1$. Blood film showed isolated thrombocytopenia. Renal function, liver function tests, chest X-ray and ultrasound of the abdomen were normal. Bone marrow aspiration showed increased megakaryocytes while other cell lines were normal. Antinuclear antibody (ANA) and dsDNA were negative. A platelet antibody test was not available. The patient had isolated thrombocytopenia without any systemic illness. A diagnosis of idiopathic thrombocytopenic purpura (ITP) was considered. The patient was started on $60 \mathrm{mg}$ prednisolone per day. The platelet count had risen to $70 \times 10^{9} / 1$ by the 10 th day and she was discharged.

In March 1983, she was re-admitted with falling platelet counts, mucosal and gingival bleeding, and epistaxis. The platelet count was $15 \times 10^{\circ} / 1$. The dose of oral steroid was increased and platelet concentrate was transfused. The platelet count did not increase and the patient developed menorrhagia and haematuria while still in the hospital. She was referred to a surgeon and, in view of the refractory thrombocytopenia, a splenectomy was performed. Postoperatively, her platelet count increased transiently for 1-2 days and then stabilised at $15-20 \times 10^{9} / 1$. She was put on danazol and cyclophosphamide after a period of 12 days postoperatively. Platelets were monitored and there was gradual but definite improvement. Six months after surgery, the platelet count was $150 \times 10^{9} / 1$ and the medicines were tapered gradually.

Between January 1984 and September 1997, she did not return for follow-up. She had eight more spontaneous abortions, six of which were in the second trimester. The cause of this was investigated. Gynaecological examination and her husband's semen analysis were normal. Abdominal ultrasound was also normal and TORCH test was negative. Her platelet count, bleeding time and clotting time were normal.

In October 1997, the patient was re-admitted with complaints of gradually increasing abdominal distension and low-grade fever. She was febrile with no pallor or lymphadenopathy. Her abdomen was distended, the umbilicus was stretched transversely and there were no dilated veins over the abdomen or back. The liver was palpable and non-tender with evidence of free fluid in the abdomen. Investigations showed normal complete blood counts including platelet count, renal and liver function tests; urine examination revealed trace proteins and chest X-ray was normal. An ascitic tap showed 150 cells, 90\% lymphocytes, and was transudative in nature. Contrast abdominal computed tomography (CT) scan (figure 1) showed gross ascites; the liver was enlarged and there was inferior vena cava (IVC) thrombosis. There were prominent retroperitoneal collateral vascular channels. On magnetic resonance angiography (MRA) (figure 2) the IVC was well visualised from its formation at the L5 level up to the L2 vertebral level. There was no

Department of Internal Medicine, Sir Ganga Ram Hospital, New Delhi 60, India

A Kakar

V Prakash

Correspondence to Dr Atul Kakar, 31 South Patel Nagar, New Delhi 10008 , India

Accepted 20 April 1999

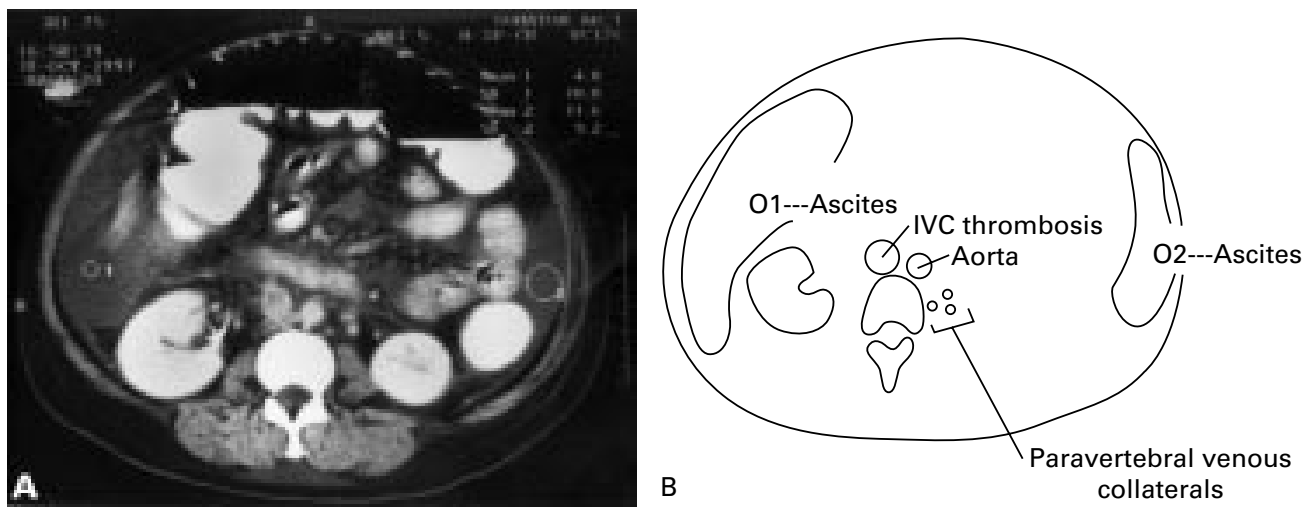

Figure 1 Contrast CT scan of abdomen showing hepatomegaly, ascites and inferior vena cava thrombosis (seen as hypodense eccentric filling defect) 


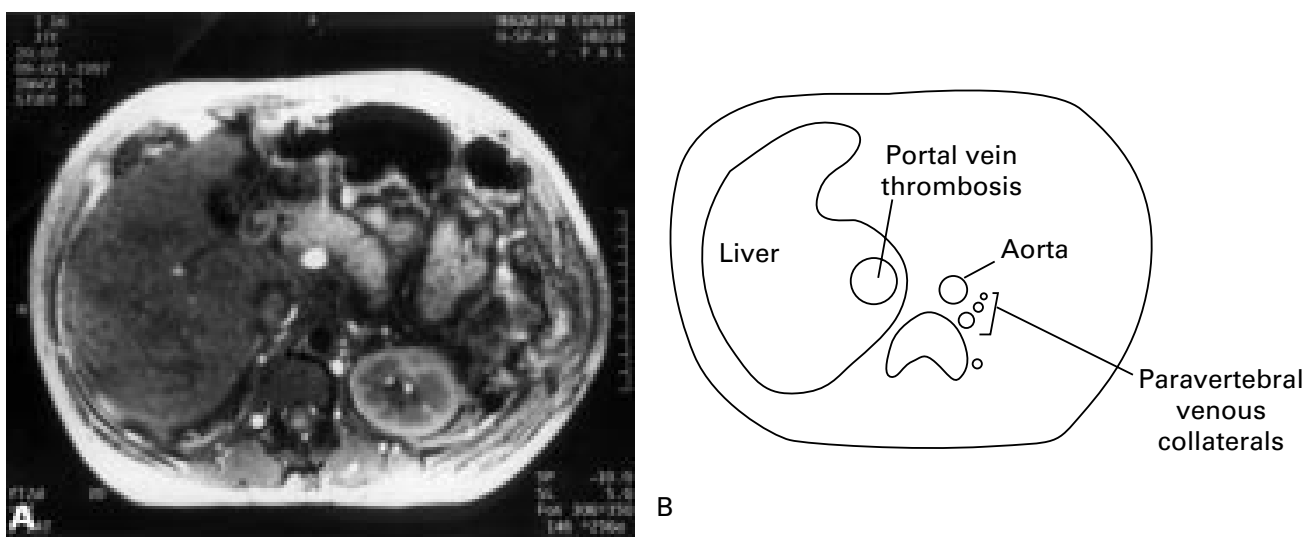

Figure 2 MRA, flash 2-D sequence, showing dark signal flow void in aorta, heterogenous slightly hyperintense signals in the intrahepatic part of the IVC due to thrombus formation. Intense bright signals are seen in portal vein and prominent paraspinal

evidence of flow intensity noted above L2 level. The flow in the inferior vena cava appeared slow and contained a central area of thrombus formation. The findings were suggestive of IVC thrombosis with collateral formation.

The patient's blood was screened for a hypercoaguable state. The activated partial thromboplastin time was $54 \mathrm{~s}$ (control $23 \mathrm{~s}$ ), lupus anticoagulant was $55.8 \mathrm{~s}$ (control $37 \mathrm{~s}$ ), anticardiolipin antibody IgG $64.413 \mathrm{~g} / 1$ (normally less than 10). The levels of protein C, S, antithrombin and fibrinogen were normal. ANA and dsDNA were both positive. Echocardiography showed pericardial effusion and 24-h urinary proteinuria was $1.2 \mathrm{~g}$.

\section{Questions}

1 What was the cause of the initial episode of severe thrombocytopenia?

2 What is the diagnosis? 


\section{Answers}

QUESTION 1

This patient had isolated thrombocytopenia with no systemic involvement. She was diagnosed as a case of ITP. Both ANA and dsDNA were negative initially.

QUESTION 2

This patient had IVC thrombosis due to antiphospholipid syndrome (APS), as both lupus anticoagulant and IgG anticardiolipin antibodies were positive. She had underlying evidence of systemic lupus erythematosus (SLE) as she had pericardial effusion, significant proteinuria, and both ANA and dsDNA were ultimately positive.

\section{Outcome}

The patient was diagnosed as having IVC thrombosis due to APS with SLE. She was started on deltaparin 5000 units bid, with prednisolone 40 $\mathrm{mg}$ /day, low-dose aspirin, and diuretics. On this treatment ascites decreased and she improved symptomatically. She was discharged on request on the 18th day of treatment on deltaparin and steroids. She discontinued the treatment on her own in the next 10 days and died 6 weeks later, probably due to pulmonary embolism.

\section{Discussion}

The triad of thrombosis (arterial and venous), foetal loss and thrombocytopenia constitutes APS. Our patient had historically all components of this syndrome but the initial event of thrombocytopenia was probably due to ITP and not to APS. An antiplatelet antibody test, if avaliable, would have supported the diagnosis. Thrombocytopenia may be the first manifestation of SLE but when it was present in our patient, there were no systemic manifestations of SLE and both ANA and dsDNA were negative. The differentiating features between thrombocytopenia in a patient with APS and due to ITP are shown in the table. In APS associated with SLE thrombocytopenia is usually mild and seldom requires treatment. ${ }^{1}$ However, our patient presented with severe thrombocytopenia which had a stormy course. Another differentiating feature is that splenectomy is not the long-term solution for thrombocytopenia in case of APS. ${ }^{2}$ Our patient had two rare coexisting diseases. The presence of these diseases simply reflect an underlying autoimmune disease. About 30\% of patients with ITP may have elevated IgG antiphosholipid antibodies at the time of diagnosis. ${ }^{3}$ Coexistent APS would increase the risk of thrombosis following splenectomy as was seen in our patient. Such patients should be given prophylactic anticoagulants. ${ }^{4}$

The term refractory ITP is applied to cases that do not respond to standard doses of steroid and splenectomy and require some other form of therapy to raise their platelet count. $^{5}$ Therapy for such patients is divided into four levels, depending on the severity of side-effects (box 1).

The treatment of acute thrombotic attack is not different from thrombosis elsewhere. Low molecular weight heparin is preferred because of a more predictable response and less incidence of thromboctyopenia. After an acute event of thrombosis, high-intensity warfarin has to be given on a long-term basis. ${ }^{2}$

It has been suggested recently that testing of lupus anticoagulant and antiphospholipid antibodies may be unnecessary for the diagnosis of ITP (box 2). ${ }^{6}$ We would conclude by

\section{Therapy used for refractory ITP \\ Level 1: prednisolone, dexamethasone, vincristine, danazole, colchicine, dapsone \\ Level 2: staphylococcal A-immunoadsorption, cyclophosphamide, azathioprine \\ Level 3: high-dose cyclophosphamide, if \\ unsuccessful then combination chemotherapy Level 4: interferon, gammaglobulin, vinblastine, cyclosporine}

Box 1

\section{Diagnosis of ITP}

- exclusion of other causes of thrombocytopenia (eg, HIV infection, SLE, lymphoproliferative disorders, myelodysplasia, drug induced, thyroid dysfunction)

- isolated thrombocytopenia with normal red cell and white cell counts and morphology

- peripheral smear showing thrombocytopenia, platelet size normal or larger than normal but giant platelets absent

- thrombocytopenia with isolated abnormality, eg, positive ANA, APS antibodies but no clinical evidence of SLE are suggestive of ITP

Box 2

Table Thrombocytopenia in ITP and APS

\begin{tabular}{lll}
\hline & ITP & APS \\
\hline $\begin{array}{ll}\text { Incidence of thrombocytopenia } \\
\text { Risk of thrombosis }\end{array}$ & All patients & None \\
Severity & Mild to severe & Yes patients \\
Clinical features & Petechiae, mucosal bleeds, menorrhagia & Usually mild \\
Antibodies directed against & Glycoprotein IIB \& IIIA or to IB-IX complex & Seldomly occur due to thrombocytopenia \\
& Exposed phospholipid on platelet membrane \\
Collagen markers & Negative & \& internal platelet antigen \\
Bone marrow & Normal or increased megakaryocytes \& precursors & Positive, except in primary type \\
& &
\end{tabular}


recommending these tests to be done in all patients with ITP at the time of diagnosis and particularly before pregnancy or any surgical procedure.

\section{Final diagnosis}

Refractory idiopathic thrombocytopenic purpura with inferior vena cava thrombosis due to

1 Martini A, Ravelli A. The clinical significance of antiphospholipid antibodies. Ann Med 1997;29:159-63.

Petri M. Pathogenesis and treatment of the antiphospholipid syndrome. Med Clin North Am 1997;81:151-73.

3 Harris EN, Ghararvi AE, Hedgde U, et al. Antiphospholipid antibodies in autoimmune thrombocytopenic purpura. $\mathrm{Br} F$ Haematol 1985;59:231-4.

4 Leuzzi RA, Davis GH, Cowchock ES, Murphy S, Vernick JJ. Management of immune thrombocytopenic purpura associated with the antiphospholipid antibody syndrome. Clin Exp Rheumatol 1997;15:197-200. antiphospholipid syndrome and systemic lupus erythematosus.

Keywords: idiopathic thrombocytopenic purpura; antiphospholipid syndrome; systemic lupus erythematosus; inferior vena cava thrombosis

5 McMillan R. Therapy for adults with refractory chronic immune thrombocytopenic purpura. Ann Intern Med 1997; 126:307-14.

6 The American Society of Haematology ITP Practice Guideline Panel. Diagnosis and treatment of idiopathic thrombocytopenic purpura: recommendations of the American Society of Haematology. Ann Intern Med 1997; 126:319-26.

\title{
Abdominal pain in a patient using warfarin
}

\author{
Javier Jimenez
}

A 32 year-old man presented with complaints of nose bleeding and mild postprandial abdominal pain for 3 days. The patient had prior history of rheumatic heart disease. Four weeks prior to the onset of symptoms the patient had undergone a double mechanical valve replacement using a number 21 Masters St Jude valve in the aortic position and a number 29 Masters St Jude valve in the mitral position. He was started on warfarin at that time. A few days prior to admission, the patient developed an upper respiratory infection and was placed on a 4-day course of azithromycin. Physical examination was unremarkable. Rectal examination revealed no gross blood, however occult blood test was positive. At the time of this visit the haemoglobin was $14 \mathrm{~g} / \mathrm{dl}$ and the International Normalised Ratio (INR) was 12. In view of these results the patient was given $5 \mathrm{mg}$ of vitamin $\mathrm{K}$ orally and sent home with close follow-up. The following day the patient was admitted to hospital with persistent abdominal pain and nausea. A repeat INR showed a level of 5. A plain abdominal X-ray was obtained, but revealed no abnormality. A few hours after admission the abdominal symptoms worsened and an abdominal computed tomography (CT) scan was ordered (figure).

Division of Cardiology, Rhode Island Hospital, Brown University Medical School, 593 Eddy Street, Providence, RI 02903, USA

J Jimenez

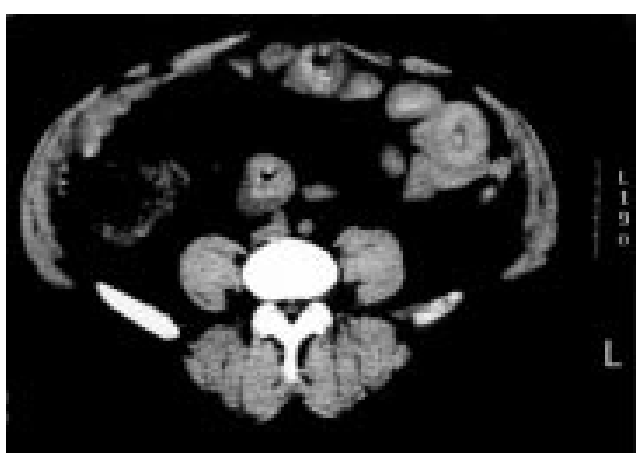

Figure Abdominal non-contrast CT scan

\section{Questions}

1 Comment on the history and CT scan. 2 How would you treat this patient? 
Answers

QUESTION 1

Oral anticoagulation is commonly used for multiple medical conditions; however, on occasions, the appropriate anticoagulation range is difficult to maintain due to patient non-compliance and/or drug interactions. Over-anticoagulation can be associated with severe bleeding complications. The abdominal non-contrast CT shows small bowel segments with areas of hyperattenuation and thickened walls. These findings, in the setting of the clinical presentation described before, are very suggestive of an intramural haematoma.

QUESTION 2

Conservative management with nasogastric suction and total parenteral nutrition can achieve resolution of obstructive symptoms. Reversal of warfarin effects is achieved acutely with vitamin $\mathrm{K}$ (10-15 mg intravenously or intramuscularly). Whole blood or fresh frozen plasma can be used to control bleeding by replacing clotting factors. Surgery should be reserved for active bleeding, presence of pneumoperitoneum, patients whose symptoms progress to an acute abdomen, or those in whom intestinal obstruction does not resolve. ${ }^{12}$

\section{Discussion}

Coumadin drug interactions are common and may affect anticoagulation levels very rapidly. Bleeding complications of over-anticoagulation may present in unusual ways. There have been several reports describing small bowel haematomas as a complication of oral anticoagulation. The incidence of intramural haematoma of the small bowel in the setting of anticoagulation is relatively rare. Bettler et al reported an incidence of 1 in 2500 patients. $^{3}$ Common medications that may increase warfarin effect are cimetidine, clofibrate, alcohol, non-

1 Gutstein DE, Rosenber SJ. Nontraumatic intramural hematoma of the duodenum complicating warfarin therapy Mt Sinai F Med 1997;64:339-41.

2 Acea Nebril B, Sanchez Gonzalez E, Aguirrezabalaga Gonzxalez J, et al. Intramural hematoma of the ileum complicating anticoagulant therapy. Rev Esp Enferm Dig 1994;86:546-9.

3 Bettler S, Montani S, Bachmann F. Incidence of intramural digestive system hematoma in anticoagulation. Epidemiologic study and clinical aspects of 59 cases observed in Switzerland (1970-1975). Schweiz Med Wochenschr 1983;113. 630 .

\section{Learning points}

- interactions with warfarin are common, namely antibiotics, alcohol, non-steroidal anti-inflammatory drugs and cimetidine

- spontaneous intramural bowel haematoma is a possible complication in patients receiving warfarin therapy

- diagnosis of intramural bowel haematoma is best performed by non-contrast abdominal CT

- intramural bowel haematoma can be treated in a conservative manner unless symptoms progress to an acute abdomen

steroidal anti-inflammatory drugs and most antibiotics, namely ciprofloxacin, erythromcyin, fluconazole, ketoconazol, metronidazole and sulfonamides.

The initial symptom of patients presenting with this complication is usually abdominal pain. Associated symptoms are nausea and vomiting. Occasionally there may also be gastrointestinal bleeding. Symptoms may progress to an acute abdomen if complete intestinal obstruction and bowel ischaemia develops. $^{4}$

Noninvasive diagnosis can be performed in most cases with non-contrast abdominal CT. ${ }^{5}$ Common findings are hyperattenuation of the involved bowel segments, with thickened bowel walls and dilated segments. Other diagnostic tools that may be used with less accuracy are abdominal X-ray films with and without contrast, and abdominal ultrasound. ${ }^{67}$

\section{Final diagnosis}

Intramural haematoma of the small bowel in the setting of warfarin anticoagulation.

Keywords: bowel haematoma; anticoagulation; warfarin

4 Azizkahn R, Poepgrass W, Wilhelm MC. Anticoaguantinduced hematomas of the small intestine. South Med $\mathcal{F}$ 1992;75:242-4.

5 Lane MJ, Katz DS, Mindelzun RE, et al. Spontaneous intramural small bowel hemorrhage: importance of non-contrast CT. Clin Radiol 1997;52:378-80.

6 Ben-Baruch D, Powsner E, Cohen M, et al. Intramural hematoma of the duodenum following endoscopic intestinal biopsy. F Pediatric Surg 1987;22:1009-10.

7 Vinard JL, Bouchet C, Aubert H, et al. Intramural hematomas of the small bowel. Report of 6 cases of which 2 required operation. $\mathcal{F}$ Chirurg 1981;118:307-14. 


\title{
A rare complication of dental abscesses
}

\author{
A Doss, P N Taylor, P F Down
}

A 27-year-old cannabis smoker was admitted with a 3-week history of gradually worsening occipital headache. He had been pyrexial one week earlier, and had been put on an oral course of amoxycillin by his general practitioner. He had a history of recurrent dental abscesses and childhood asthma. He was not an intravenous drug abuser.

On examination he was well with a temperature $37.5^{\circ} \mathrm{C}$, pulse rate 80 beats $/ \mathrm{min}$, blood pressure 130/80 mmHg. He had bilateral papilloedema, sustained ankle clonus, and brisk knee and ankle jerks. Examination was otherwise unremarkable. Full blood count, apart from a mean corpuscular volume of $101 \mathrm{fl}$, was normal. Liver function tests, urea, electrolytes, clotting, thrombophilia screen, auto-immune profile, erythrocyte sedimentation rate, serum electrophoresis and blood cultures were unremarkable. Visual field testing showed slightly enlarged blind spots in both eyes. An urgent brain computed tomography (CT) scan was performed within (figure 1).

Two days following admission he developed pain and swelling of the right lower jaw and was referred to the maxillofacial surgeons who diagnosed dental abscesses. He went on to have incision and drainage of the lower right buccal space and multiple dental extractions under general anaesthesia. He was treated with antibiotics for 2 weeks and discharged home after making a full recovery. $\mathrm{He}$ is currently under follow-up with monthly visual field testing and regular dental review.
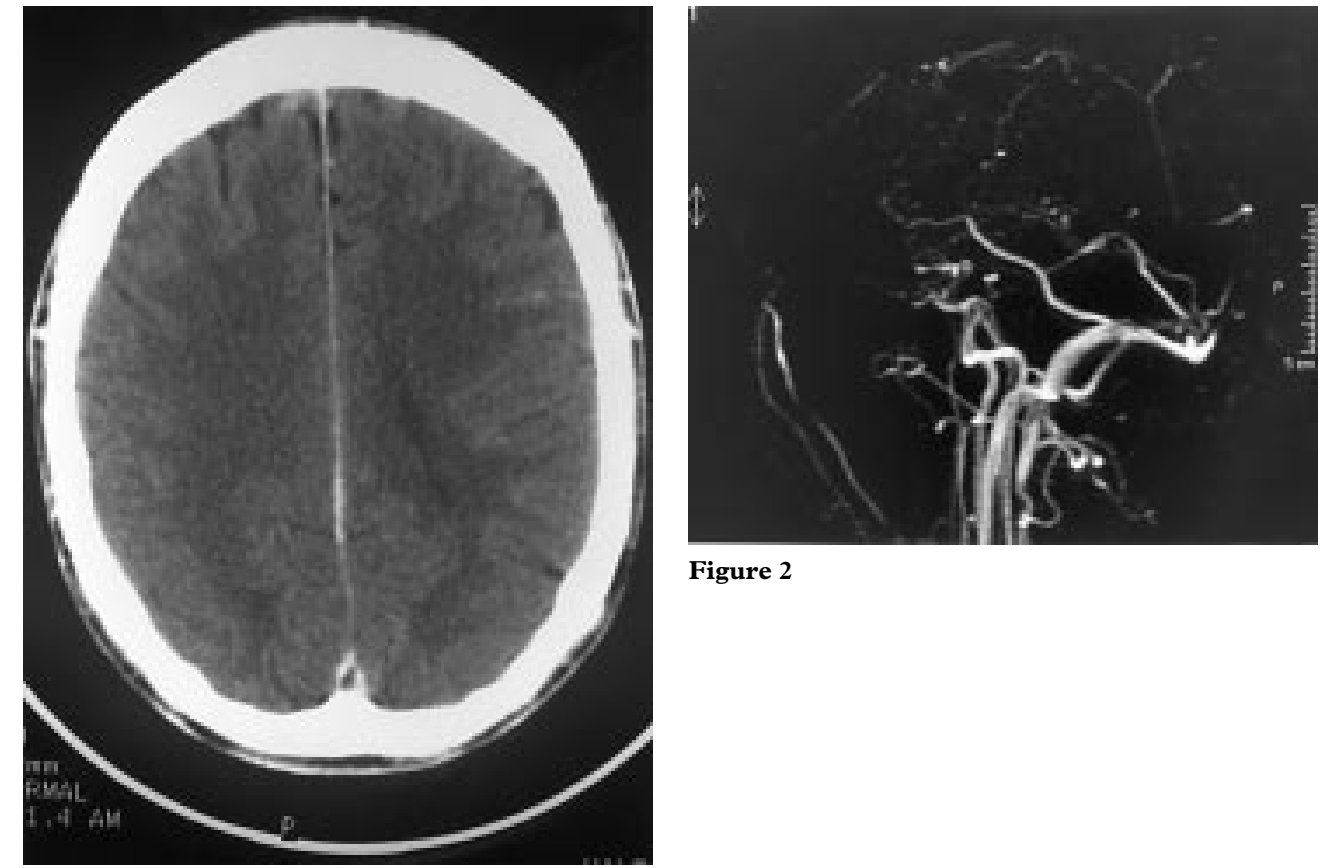

Figure 2
Department of Medicine, Dorset County Hospital, Dorchester, Dorset, UK

A Doss

P N Taylor

P F Down

Correspondence to A Doss, Department of Diagnostic Radiology, Royal Hallamshire Hospital, Sheffield, UK

Accepted 20 April 1999

\section{Questions}

1 What does figure 1 show? What is this sign called?

2 What is the investigation shown in figure 2 ? What does it show?

3 Is there an association between the CT findings and the dental abscesses?

4 Are anticoagulants routinely indicated in this condition? 
Answers

QUESTION 1

There is a filling defect in the superior sagittal sinus in keeping with superior sagittal sinus thrombosis (figure 1). This appearance is known as the 'delta sign'. On an enhanced brain CT scan, the normal sagittal sinus is homogenously opaque.Thrombus within the lumen of the sinus appears as a filling defect outlined by contrast, often triangular or deltashaped, which is known as the delta sign.

QUESTION 2

Figure 2 is a phase-contrast magnetic resonance angiography (MRA) sequence coded for venous flow. This confirms occlusion of the superior sagittal sinus.

QUESTION 3

It is possible that dental abscesses played a causative role in the sagittal sinus thrombosis of our patient. Septic intracranial sinus thrombosis as a complication of upper respiratory tract infections is well recognised.

QUESTION 4

Anticoagulation is not routine in sagittal sinus thrombosis. Management relies on treating the underlying cause and raised intracranial pressure rapidly and effectively.

\section{Discussion}

As far as we know this is the first case report of septic sagittal sinus thrombosis associated with dental abscesses. Sepsis is a predisposing factor, most frequently bacterial meningitis or facial sinus infection. ${ }^{1}$ Other reported associations include primary thrombocythaemia, homocystinuria, intracranial angiography, dehydration, Behcet's disease, haemolytic anaemia, coagulopathies, inhalational drug abuse, the post-partum period and the oral contraceptive pill. $^{2}$ The natural history of this condition is highly variable with mortality ranging between $10-20 \%$.

At present, venous MRA is probably the definitive examination and the gold standard for diagnosis of dural sinus thrombosis. ${ }^{3}$ How-

1 Southwick FS, Richardson EP Jr, Swartz MN. Septic thrombosis of the dural venous sinuses. Medicine (Baltimore) thrombosis of the

2 Mohammed A, McLeod JG, Hallinan J. Superior sagittal sinus thrombosis. Clin Exp Neurol 1991;28:23-36.

3 Cipri, S, Gangemi A, Campolo C, Cafarelli F, Gambardella G. High dose heparin plus warfarin administration in non-traumatic dural sinuses thrombosis. A clinical and neuroradiological study. F Neurosurg Sci 1998;42:23-32.

4 Thron A, Wessel K, Linden D, Schroth G, Diehgans J. Superior sagittal sinus thrombosis: neuroradiological evaluation and clinical findings. F Neurol 1986;233:283-8.

\section{Sagittal sinus thrombosis}

- septic complication of bacterial meningitis, facial sinus infection, dental abscess

- $10-20 \%$ mortality, worse in sepsis

- diagnosis: cranial CT with contrast ('delta sign'); MRA is the definitive investigation

- treatment is of underlying condition and raised intracranial pressure; anticoagulation is controversial; direct thrombolysis is increasingly used

- other associations: post-partum, dehydration, marasmus, oral contraceptives, inhalational drug abuse, coagulopathies, haemolytic anaemia, primary thrombocythaemia, sickle cell anaemia, Behcet's disease, head injury, homocystinuria

ever, brain CT, which is usually the initial examination, may be diagnostic by demonstrating the 'delta sign' in $70 \%$ of cases. ${ }^{4} \mathrm{CT}$ or MRI may also identify areas of haemorrhage or venous infarction in the adjacent brain.

The management of intracranial dural sinus thrombosis is still controversial and uncertain. There are no controlled trials of therapy. However, it is important to treat the underlying cause and raised intracranial pressure. Anticoagulants may be indicated early when there is no radiological evidence of haemorrhage. ${ }^{23}$ More recently, direct thrombolysis of dural sinus thrombosis has shown a better outcome in these patients. ${ }^{56}$

The decision regarding anticoagulation should be based on the severity of acute presentation, underlying associated conditions, evidence of haemorrhage or venous infarction of adjacent brain tissue on CT or MRI, and advice from neurologists and neurosurgeons should be sought sooner rather than later.

\section{Final diagnosis}

Superior sagittal sinus thrombosis as a complication of dental abscesses.

Keywords: superior sagittal sinus thrombosis; dental abscess; papilloedema; anticoagulation

\footnotetext{
5 Niwa J, Ohyama H, Matumura S, Maeda Y, Shimizu T. Treatment of acute superior sagittal sinus thrombosis by t-PA infusion via venography-direct thrombolytic therapy in the acute phase. Surg Neurol 1998;49:425-9.

6 Kuether TA, O’Neill O, Nesbitt GM, Barnwell SL Endovascular treatment of traumatic dural sinus thrombosis; case report. Neurosurgery 1998;42:1163-7.
} 


\title{
Abnormal skull X-ray in a child with growth retardation
}

\author{
A S Kashyap
}

A 41-month-old female child was referred to the endocrine clinic for evaluation of poor growth, and delayed motor and mental milestones. She was the only child born out of a non-consanguineous marriage. There was no history of hypothyroidism or goitre in the parents. There was no family history of congenital hypothyroidism. Clinical evaluation revealed a lethargic child with dry skin, open anterior fontanelle, protuberant abdomen and short limbed dwarfism. Her bone age was less than 2 years and height age was 20 months. Her skull X-ray is shown in the figure.

Department of

Medicine, Armed

Forces Medical

College, Pune 411040,

India

A S Kashyap

Accepted 26 April 1999

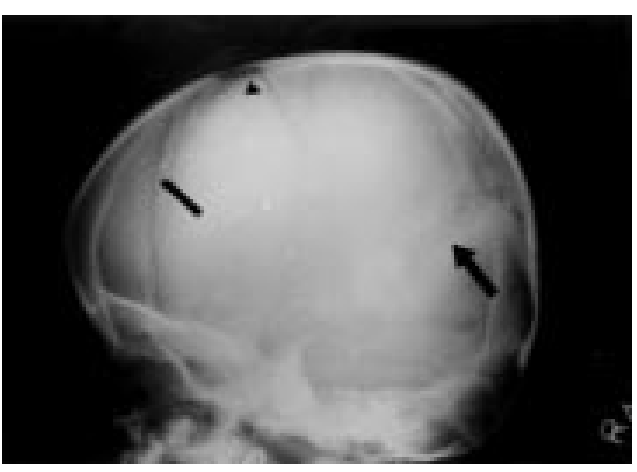

\section{Questions}

1 What is the diagnosis?

2 What abnormalities are shown in the skull $\mathrm{X}$-ray?

3 What other skeletal abnormalities are seen in this condition? 
Answers

QUESTION 1

This patient has congenital hypothyroidism. Detailed clinical evaluation revealed, in addition to the above findings, an enlarged tongue, coarse facial features, generalised hypotonia, hoarse cry, and delayed relaxation of tendon jerks. There was no goitre. Serum thyroidstimulating hormone levels were $51 \mathrm{mU} / 1$ (normal $0.5-5 \mathrm{mU} / 1$ ), serum thyroxine levels were $10 \mathrm{nmol} / 1$ (64-154 nmol/l) and serum triiodothyronine $\left(\mathrm{T}_{3}\right.$ levels were $0.2 \mathrm{nmol} / 1(1.1-$ $2.9 \mathrm{nmol} / \mathrm{l})$. Sodium [ $\left.{ }^{99 \mathrm{~m}} \mathrm{Tc}\right]$ pertechnetate scintiscanning revealed negligible uptake in thyroid region. This investigation profile is consistent with thyroid dysgenesis or agenesis leading to sporadic congenital hypothyroidism. Congenital hypothyroidism has an incidence of 1 in $3500-4000$ and is easily picked up by neonatal screening. If diagnosed and treated at an early stage the manifestations of hypothyroidism can be reversed and/or prevented.

QUESTION 2

The skull X-ray displays intrasutural or wormian bones along lambdoid (broad arrow) and coronal sutures (narrow arrow). The anterior fontanelle is visualised (delayed closure of anterior fontanelle, arrow head). These are typical skull $\mathrm{X}$-ray findings in congenital hypothyroidism. ${ }^{1}$ Wormian bones disappear when bone age reaches 5 years. These bones may also be seen in cleidocranial dysplasia, pyknodysostosis, acro-osteolysis (HajduCheney syndrome) and osteogenesis imperfecta.

In congenital primary hypothyroidism, the skull may be brachycephalic (a result of endochondral growth retardation at the base of skull). The sella is small and bowl shaped in young children and larger and rounded (cherry sella) in older children due to rebound hypertrophy of the pituitary gland. The paranasal sinuses are underdeveloped and facial bones are hypoplastic. An increased thickness of bones of the cranial vault with narrow diploic space may be seen. Development of teeth is delayed; primary teeth remain for several years beyond the normal time for exfoliation. Comparable delay occurs in appearance of permanent teeth. Unerupted teeth are structurally abnormal and are subject to caries. Dental defects tend to parallel the delay in ossification of the skeleton. The poorly developed jaw shows gross dental crowding.

Epiphyses are retarded in appearance and closure. When ossification or epiphyseal dysgenesis does occur, it is often from multiple sites within the epiphysis. This leads to a spotted or fragmented appearance on X-ray. This feature is most commonly seen in femoral and humeral heads, and in the navicular bone of the foot. Epiphyseal disturbances, particularly in the femoral head, persist beyond a bone age of 8 years. Femoral epiphyseal dysgenesis may resemble Perthe's disease, though Perthe's disease is usually unilateral. The incidence of slipped capital femoral epiphysis is increased in these cases of hypothyroidism. ${ }^{2}$ The long bones
Bone and skeletal manifestations of hypothyroidism

Skull

- delayed closure of fontanelle

- relatively large sella

- poorly developed paranasal sinuses

- brachycephaly

- delayed dentition and dental caries

- wormian bones

Skeleton

- dwarfism

- increased density

Epiphyseal centres of ossification

- retarded growth

- multicentric and irregular

- delayed fusion and stippled appearance

- epiphyseal dysgenesis (fragmented epiphyses)

Spine

- kyphosis

- flattening of vertebral bodies

- increased width of intervertebral space

- bullet-shaped vertebral bodies (usually L-1 and

L-2)

Long bones

- short-limbed dwarfism

- dense transverse bands at metaphyseal ends

Pelvis

- narrow with coxa-vara

are short and this leads to disproportionate (short limbed) dwarfism. Dense transverse bands at metaphyseal ends may be present very early in life, but they tend to disappear by a bone age of 6 months. The pelvis is often narrow with coxa-vara deformity.

In severe involvement, bullet-shaped vertebrae are seen (usually T-12 or L-1). This is due to some degree of flattening of vertebral bodies with forward slipping of one vertebra over another, resulting in thoracolumbar gibbus. Disc spaces may be widened. Diffuse osteoporosis of vertebral bodies leads to the appearance of 'picture framing' of vertebral bodies. Increased density of the skeleton is seen in some cases.

\section{Discussion}

The thyroid hormone is important for regulation of normal growth, development and maturation of tissue. In hypothyroidism pathological changes include a marked decrease in cartilage cell proliferation. The osseous tissue abuts the cartilage zone; this forms a barrier to longitudinal growth of the bone. Growth failure is due to both impaired protein synthesis and reduction in insulin-like growth factor-1 levels. ${ }^{3}$ The decrease in protein synthesis is reflected in retardation of skeletal and soft tissue growth. Thyroid hormone deficiency thus impairs secretion as well as effectiveness of growth hormone.

Thyroid hormone effects on bone growth are direct and indirect. Proof of direct effect of thyroid hormones on bone growth needs to be demonstrated by thyroid receptors in bone cells and responses to thyroid hormones in 
vitro. Osteoclasts and osteoblasts have been shown to have a specific in-vitro response to $\mathrm{T}_{3}$. Little is known about the expression of thyroid receptor (TR) in developing bone and cartilage. In studies of osteosarcoma cells, expression of TR beta mRNA was greater than TR alpha mRNA in mature osteoblasts, whereas the opposite was true in fibroblast precursors of osteoblasts. ${ }^{4}$ This provides support for bone responsiveness to $\mathrm{T}_{3}$. Retinoid $\mathrm{X}$ receptor, a cofactor involved in the interaction of TR with $\mathrm{T}_{3}$-responsive element, has also been found to be expressed in osteoblasts. ${ }^{5}$

Thyroid hormone influences bone development and growth indirectly via growth hormone $(\mathrm{GH})$ secretion and action. GH secretion and synthesis are stimulated in vivo by thyroid

1 Vogler JB, Genant HK. Metabolic and endocrine disease of the skeleton. In: Grainger RG, Allison D, eds. Grainger and Allison's Diagnostic radiology: A textbook of medical imaging. 3rd edn. 1997; p 1302

2 Crawford AH, MacEwen GD, Fonte D.Slipped capital femoral epiphyses coexistent with hypothyroidism. Clin Orthop 1977;122:35-140.

3 Cavaliere H, Knobel M, Medeiros-Neto G. Effect of thyroid hormone therapy on plasma insulin-like growth factor 1 levels in normal subjects, hypothyroid patients and endemic cretins. Horm Res 1987;25:132-9. hormone. This effect is due to direct interaction of TR-T ${ }_{3}$ complex with $\mathrm{GH}$ gene regulating its expression. Thyroid hormone treatment has been reported to more than double the nocturnal $\mathrm{GH}$ values in hypothyroid children. ${ }^{6}$ Reversal of blunted $\mathrm{GH}$ response to $\mathrm{GH}-$ releasing hormone stimulation in hypothyroid children following treatment with thyroid hormone indicates a direct influence of thyroid hormone on $\mathrm{GH}$ secretion at the level of the pituitary rather than the hypothalamus.

\section{Final diagnosis}

Congenital hypothyroidism.

Keywords: wormian bones; hypothyroidism

4 Williams G, Bland R, Sheppard M. Characterisation of thyroid hormone [T3] receptors in three osteosarcoma cell lines of distinct osteoblast phenotype: interactions among T3, vitamin D3 as retinoid signaling. Endocrinology 1994;35: vitamin

5 Kindmark A, Torma H, Johansson A, et al. Reverse transcription-polymerase chain reaction assay demonstrates that the 9-cis retinoic acid receptor alpha is expressed in human osteoblasts. Biochem Biophys Res Commun 1993;192:1367-72. 6 Chernausek S, Underwood L, Utiger R, et al. Growth hormone secretion and plasma somatomedin- $\mathrm{C}$ in primary hypothyroidism. Clin Endocrinol 1983;19:337-9.

\title{
A respiratory complication of diabetic ketoacidosis
}

\author{
N Younis, M J Austin, I F Casson
}

A 37-year-old man with type 1 diabetes presented with a few days history of persistent vomiting and lethargy associated with thirst and polyuria. He was not on any regular medication apart from insulin. He had omitted his insulin over the last 24 hours.

Clinical examination revealed him to be dehydrated with a tachycardia of 120 beats $/ \mathrm{min}$ and blood pressure $130 / 80 \mathrm{mmHg}$. He was dyspnoeic with a respiratory rate of $32 \mathrm{breaths} / \mathrm{min}$; the pattern was characteristic of Kussmauls respiration. Laboratory investigations showed a metabolic acidosis with arterial blood gases $\mathrm{pH} 7.08$, bicarbonate $10.7 \mathrm{mmol} / \mathrm{l}$, base excess -22.6 $\mathrm{mmol} / \mathrm{l}$ and plasma glucose $32.4 \mathrm{mmol} / \mathrm{l}$. Ward testing for urinary ketones was strongly positive (+++ by ketostix). A chest X-ray was performed (figure).

\author{
Department of \\ Diabetes and \\ Endocrinology, Royal \\ Liverpool and \\ Broadgreen University \\ Hospital, Liverpool, \\ UK \\ $\mathrm{N}$ Younis \\ M J Austin \\ I F Casson
}

Accepted 28 April 1999

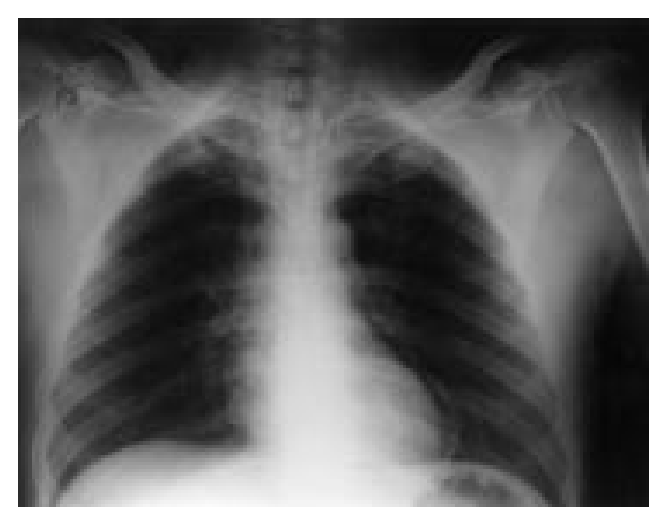

\section{Questions}

1 What does the chest X-ray show?

2 What clinical signs would you look for? 
Answers

QUESTION 1

The chest X-ray shows mediastinal emphysema with characteristic lines of radiolucency around the mediastinal pleura. There is also radiological evidence of subcutaneous emphysema in the soft tissues in the neck.

QUESTION 2

Surgical emphysema is frequently palpable in the neck and may be more widespread involving the face, chest or arm. Hamman's sign is variously described as a crepitous, crackling or crunching sound, synchronous with systole that may be heard with the stethoscope. Pleural effusion may accompany mediastinal emphysema caused by oesophageal rupture.

\section{Discussion}

Pneumomediastinum is a well recognised but infrequent complication of diabetic ketoacidosis. ${ }^{1-4}$ The incidence of this complication is unknown. In most but not all reports there is a history of persistent or severe vomiting. ${ }^{13}$ The prognosis is excellent and there is prompt regression of the pneumomediastinum following correction of the ketoacidosis. ${ }^{1-3}$ It is thought that in diabetic ketoacidosis hyperventilation induced by acidosis or by severe vomiting causes changes in the intra-alveolar pressure gradient within the lungs. ${ }^{5}$ The rise in intra-alveolar pressure causes rupture and subsequent dissection of air escaping alongside perivascular sheaths into the mediastinum; air enters into fascial planes particularly in the neck causing subcutaneous

1 Mc Nicholl B, Murray JP, Egan B, McHugh P. Pneumomediastinum and diabetic hyperpnoea. BMF 1968;4:493-4.

2 Meeking DR, Krentz A J. Pneumomediastinum complicating diabetic ketoacidosis. Diabet Med 1996;13:587-8.

3 Bullaboy CA, Jennings RB Jr, Johnson DH, et al. Radiological case of the month. Pneumomediastinum and subcutaneous emphysema caused by diabetic hyperpnea. Am $\mathcal{F} D i$ s Child 1989;143:93-4.
Pulmonary abnormalities in patients with diabetes mellitus

Infections

- zygomycosis (mucormycosis)

- mycobacterioses

- bacterial and viral infections

Physiological changes

- reduced elastic recoil of the lungs

- reduced diffusion capacity of the lungs for carbon monoxide

- diminished bronchial reactivity

- elevated arterial oxygen saturation

- elevated arterial oxygen tension

- disordered breathing patterns: central hypoventilation, sleep apnoea

Others

- pulmonary oedema

- aspiration pneumonia

- pulmonary xanthogranulomatosis

- pneumomediastinum and pneumothorax

emphysema. ${ }^{5}$ Our patient had no specific symptoms to suggest the diagnosis of pneumomediastinum. Thus, unless a chest $\mathrm{X}$-ray is performed, the diagnosis can be missed. Other recognised pulmonary complications of diabetes mellitus are listed in the box.

\section{Final diagnosis}

Pneumomediastinum as a complication of diabetic ketoacidosis.

Keywords: pneumomediastinum; emphysema; ketoacidosis; diabetes

4 Grieve NW, Bird DR, Collyer AJ, Meredith GA. Pneumomediastinum and diabetic hypernoea (letter). BMF 1969;1: 186.

5 Macklin MT, Macklin CC. Malignant intersitial emphysema of the lungs and mediastinum as an important occult complication in many respiratory diseases and other cons: 


\title{
An unusual case of transient ischaemic attacks
}

\author{
B R Archana
}

A 29-year-old man, with no significant history, presented with a 2-month history of episodic facial numbness on the left side associated with left-sided weakness and expressive dysphasia, lasting about 1 minute. There were no visual disturbances. He was a martial arts enthusiast and led a very active life. He denied any recent trauma to his head or neck. He was a non-smoker and consumed less than 2 units of alcohol a week.

Examination revealed a young man with a pulse rate of 90 beats $/ \mathrm{min}$, and blood pressure of $120 / 80 \mathrm{mmHg}$. There was no rash, petechiae or lymphadenopathy. Thoracic, cardiovascular and abdominal examinations were normal. No carotid bruits were heard. Nervous system examination revealed normal higher mental functions and no cranial nerve involvement. Motor examination was normal except for an equivocal left plantar response. Sensory examination revealed a patch of altered sensation over the left side of the face. The optic fundi were normal.

The results of the routine laboratory investigations revealed a haemoglobin of $15.1 \mathrm{~g} / \mathrm{dl}$, and glucose of $5.7 \mathrm{mmol} / 1$. Electrolytes were within the normal range. Computed tomography of the brain appeared normal. An electroencephalogram showed intermittent slow wave activity in the right anterior and temporal regions. Magnetic resonance imaging (MRI) of the brain (figure 1) revealed multiple areas of high attenuation in the corona radiata and right temporal regions suggestive of ischaemia. A further investigation was performed (figure 2).

Frenchay Hospital, Frenchay, Bristol BS16 1LE, UK

B R Archana

Correspondence to BR Archana, \#22, Flat 7 , Clark Hall, Frenchay Hospital, Frenchay, Bristol BS16 1LE, UK

Accepted 14 May 1999

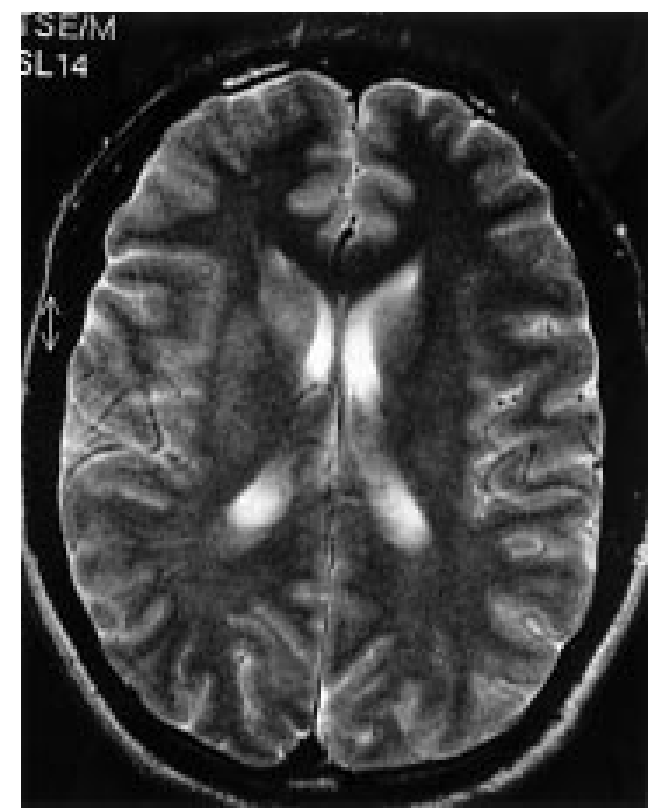

Figure 1 Brain MRI

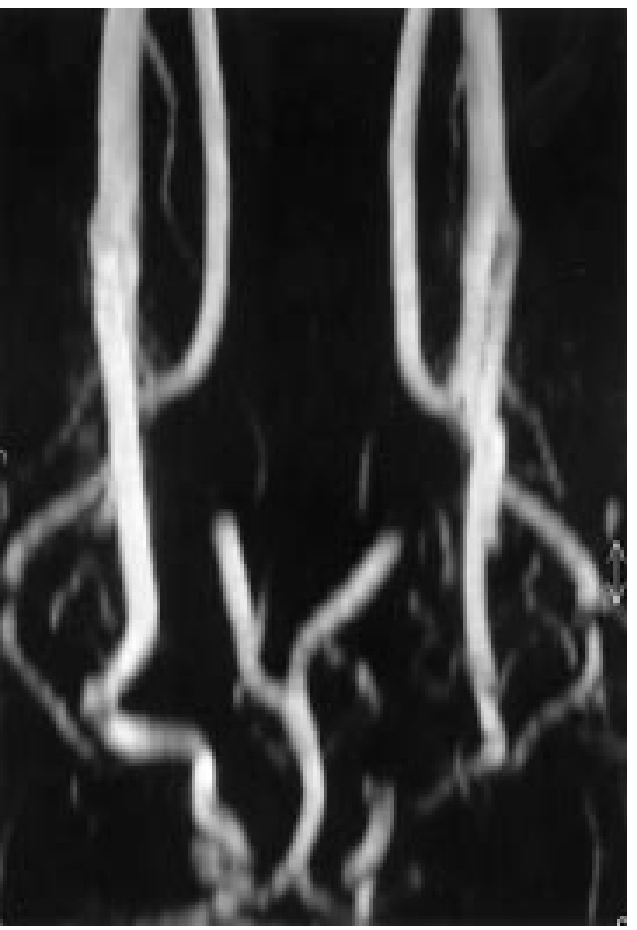

Figure 2

\section{Questions}

1 What investigation is shown in figure 2 and what does it suggest?

2 What further investigations are appropriate?

3 What is the management and prognosis? 


\section{Answers}

QUESTION 1

Magnetic resonance angiography suggesting bilateral carotid artery dissection.

QUESTION 2

Carotid Doppler studies confirmed the findings. Echocardiography revealed a normal heart.

QUESTION 3

Anticoagulant treatment is often used for a few months when the dissection involves the extracranial segment of the carotid artery. The prognosis of carotid dissections depends on the presence and severity of ischaemic brain damage. Normalisation of the vascular abnormalities is frequent and is an excellent argument in favour of the prognosis.

\section{Discussion}

Arterial dissection results from bleeding into the vessel wall. Some cases are associated with cervical trauma or have evidence of underlying vascular disease. In the above case, strain on the neck due to martial art manoeuvres might have been the cause. The extracranial segment

1 Mas JL. Internal artery dissection. Rev Pract 1993;43:50914. of the internal carotid artery is the vessel most commonly involved. Intracranial carotid dissections are much rarer. Carotid dissection occurs predominantly in young or middle aged adults and shows no sex predominance. Although clinical manifestations can range from isolated headache to rapidly lethal stroke, the most common and suggestive syndrome associates 'local' symptoms (such as head or neck pain, Horner's syndrome, pulsatile tinnitus or cranial nerve palsies) and delayed symptoms of cerebral ischaemia in the territory of the internal carotid artery. Dissection can be bilateral or associated with dissection of the vertebral artery. Angiography has long been considered the gold standard for the diagnosis. As this procedure carries a risk of cerebral complications, noninvasive diagnostic approaches such as MRI and ultrasound are being increasingly used. ${ }^{2}$ Intra-arterial angiography is no longer necessary.

\section{Final diagnosis}

\section{Bilateral carotid artery dissection.}

Keywords: carotid artery dissection; transient ischaemic attacks

\footnotetext{
2 Auer D, Karnath HO, Nagele T, Dichgans J . Non invasive investigations of pericarotid syndrome: role of MR angioinvestigations of pericarotid syndrome: role of MR angio-
graphy in the diagnosis of internal carotid dissection. Headache 1995;35:163-8.
} 
University of

Massachusetts Medical

Center, 55 Lake

Avenue North,

Worcester, MA 01655

USA

Department of

Neurology

N Selvaraj

D A Drachman

P Ravin

Department of

Radiology

(Neuroradiology)

J R Knorr

Accepted 14 May 1999

\title{
Myocardial infarction, seizure and an abnormal head CT scan
}

\author{
Nithianandam Selvaraj, David A Drachman, Paula Ravin, John R Knorr
}

A 60-year-old woman was admitted with acute substernal chest pain. Electrocardiographic findings and creatine kinase values were consistent with an acute anteroseptal myocardial infarction. She underwent a coronary angiogram and percutaneous transluminal coronary angioplasty (PTCA) with stent placement. Two hours following the procedure, the patient developed rightsided partial motor seizures with secondary generalisation. A head computed tomography (CT) scan was obtained immediately to rule out a stroke.

Her medical history was significant for coronary heart disease, hypertension, asthma and seizures. Her last seizure was almost 10 years ago. She had taken an anti-epileptic medication in the past and had stopped it on her doctor's advice. There was also a remote history of alcohol and intravenous drug abuse. Prior to this admission, she was taking zestril, metoprolol, bupropion for smoking cessation, premarin, and steroid inhalers. After admission, she received aspirin, ticlid, intravenous nitroglycerin and abciximab (ReoPro). Abciximab was started during her PTCA.

When examined four hours after her seizure, she was alert and oriented. Her speech and language functions were normal. Cranial nerve examination did not reveal any abnormalities. On motor testing, she was noted to have a right pronator drift. Her muscle strength was entirely normal. Sensation was preserved throughout. Muscle stretch reflexes were $2+$ and symmetrical. Babinski sign was present on the right side. The left plantar reflex was normal. Coordination was intact. No signs of meningeal irritation were present.

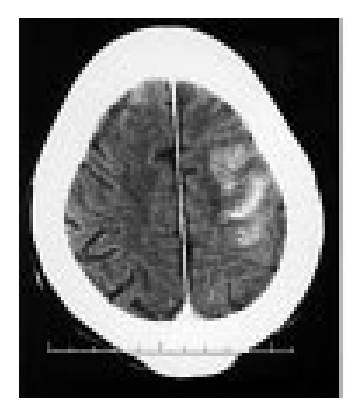

Figure 1 CT scan

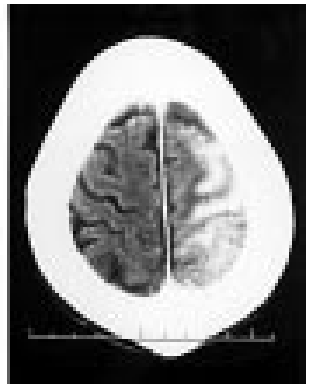

Questions

1 What is the radiological diagnosis, taking into account the patient's hospital course?

2 What are the possible aetiologies of her seizure?

3 What is the further management? 


\section{Answers}

QUESTION 1

The CT scan of the head shows hyperdensity involving the left cerebral hemisphere. Although this was meant to be an unenhanced $\mathrm{CT}$, the fact that the patient had received a contrast agent (Hypaque) for her coronary angiography makes this CT, in fact, a contrastenhanced study. It is important to recognise this when interpreting the abnormal findings. To the inexperienced eye this may look like subarachnoid haemorrhage or a haemorrhagic cerebral infarction. On close examination, however, it is evident that the hyperdensity involves mainly the white matter rather than filling the sulci (see figure 2).
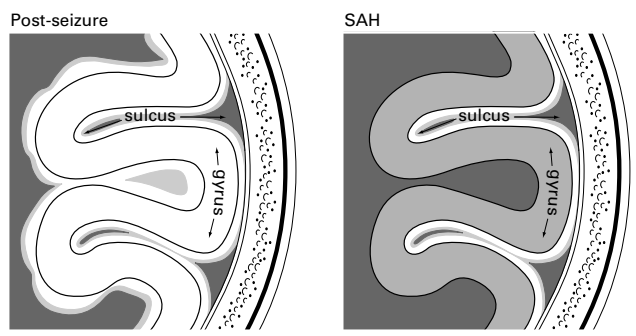

Figure 2 As shown in this schematic diagram, the CT appearance in our patient is not subarachnoid haemorrhage (SAH) because the hyperdensity mainly occupies the gyri and not the sulci

The absence of sulcal enhancement and the absence of blood in the interpeduncular cistern makes the diagnosis of subarachnoid haemorrhage unlikely. As the lesion does not correspond clearly to a vascular territory and has both diffuse white matter and cortical involvement, the diagnosis of a haemorrhagic infarct is less likely. Furthermore, the radiographic findings are out of proportion to the patient's relatively normal neurological status. This also has to be taken into account when interpreting the abnormal CT findings.

QUESTION 2

The aetiology of the seizures in this patient is unclear. The differential diagnosis is shown in the box.

\begin{tabular}{|l|}
\hline Differential diagnosis \\
\hline - contrast medium-induced seizure \\
- relapse of a pre-existing seizure disorder \\
- bupropion-induced seizures \\
\hline
\end{tabular}

The patient had a history of generalised seizures many years ago. She has been seizure-free for at least 10 years without any medications; in the past seizures may have been related to alcohol withdrawal. Hence, it is unclear if this seizure was related to the history of seizures.

Bupropion is known to cause seizures in $0.1-0.4 \%$ of patients taking the drug. The risk in patients with a history of seizures may be even higher. We do not know if the drug was causally related to the current episode.

Abciximab (ReoPro) is being used increasingly in cardiac patients. It is the Fab fragment of the chimeric human-murine monoclonal antibody $7 \mathrm{E} 3$. It binds to the glycoprotein $\mathrm{IIb} /$ IIIA receptor of human platelets and inhibits platelet aggregation. It is used as an adjunct to PTCA for the prevention of acute cardiac ischaemic complications. Nervous system sideeffects reported include cerebral ischaemia $(0.3 \%)$ and coma $(0.4 \%)$. Although it is theoretically possible that cerebral ischaemia can predispose to seizures in patients taking abciximab, seizures have not been reported with the use of this drug.

Neurotoxicity from intravascular contrast, in particular the ionic high-osmolar agents has been described previously. Contrast mediuminduced seizures are also well described in the literature and it is probably the most likely aetiology for seizures in our patient, although a combination of mechanisms is possible.

\section{QUESTION 3}

One reasonable approach would be to monitor the patient clinically and if her neurological status did not change, repeat a non-contrast CT in 24 hours. In our patient, a non-contrast CT repeated the next day (figure 3) was entirely normal.

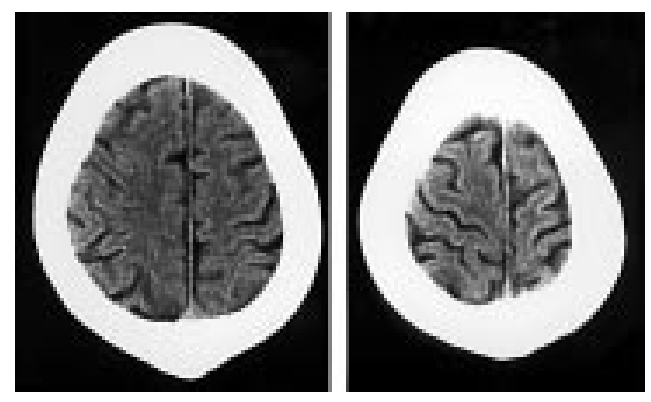

Figure 3 Non-contrast CT scan

The hyperdensity noted in the initial CT had completely disappeared. The minor residual neurological deficits noted post-ictally (Todd's paralysis) completely resolved over 24 hours. As the CT changes were unilateral, we presumed that the ictal activity originated from the left hemisphere. However, an electroencephalogram (EEG) obtained to look for a left hemispheric focus was completely normal. The patient was discharged home after her cardiac status was stable. Phenytoin treatment was begun as the aetiology of her seizures was unclear, pending further evaluation. 


\section{Discussion}

Contrast-enhanced CT scans of the brain obtained on patients soon after seizures are often abnormal. The changes on CT may be either hypodensities or hyperdensities. Various authors have described reversible radiographic signs after seizures. ${ }^{1-3}$ All these changes typically disappear over time, most within 1-2 weeks. The underlying mechanism is believed to be a transient disruption of the blood-brain barrier (BBB).

In vitro studies have shown that both electrically and pharmacologically induced seizures cause transient breakdown of the BBB. ${ }^{4}$ During prolonged seizure activity, BBB disruption leads to increased water content in brain tissue (cerebral oedema), especially in the hypothalamus and hippocampus. Both local and systemic changes account for the cerebral oedema. Relative hypoxia due to increased ictal metabolism, with an increase in local $\mathrm{pCO}_{2}$ and lactate, results in local vasodilatation and loss of autoregulation. ${ }^{5}$ Systemically there is an increase in blood pressure and decrease in blood $\mathrm{pH}$, again resulting in decreased cerebral autoregulation and local hyperaemia. ${ }^{6}$ It has also been reported that the degree of disruption of the $\mathrm{BBB}$ is proportional to the duration of seizure activity. Systemic hypertension during seizures, in combination with disrupted $\mathrm{BBB}$, can lead to cerebral oedema, causing hypodense lesions and, in the presence of contrast agents, abnormal-appearing hyperdensities on CT scanning.

Sage and colleagues have demonstrated increased permeability of the BBB after large intracarotid contrast injections. ${ }^{7}$ Scott has previously reported that intravenous administration of contrast material for head CT may precipitate generalised seizures, especially in cases of metastatic neoplasm of the brain. ${ }^{8}$

1 Goulatia RK, Verma A, Mishra NK, et al. Disappearing CT lesions in epilepsy. Epilepsia 1987;28:523-7.

2 Clarke HB, Gabrielson TO. Seizure induced disruption of the Blood brain barrier demonstrated by CT. F Comput Assist Tomogr 1989;13:889-92.

3 Kramer RE, Luders H, Lesser RP, et al: Transient focal Kramer RE, Luders $\mathrm{H}$, Lesser RP, et al: Transient foca
abnormalities of neuroimaging studies during focal status abnormalities of neuroimaging studies

4 pee JC, Olszewski J. Increased cerebrovascular permeability after repeated electroshocks. Neurology 1961;11:515-9.

5 Lee SH, Goldberg HI. Hypervascular pattern associated

5 Lee SH, Goldberg HI. Hypervascular pattern associated with idiopat

6 Plum F, Posner JB, Troy B. Cerebral metabolic and circulatory responses to induced convulsions in animals. Neurology 1968;18:13.
Neurologic complications after cardiac catheterisation are well known. Contrast agent neurotoxicity presenting with focal seizures with CT head findings similar to subarachnoid haemorrhage has also been reported. ${ }^{9}$ Contrastmedium-induced seizures are well described in the literature and their incidence has been reported to be about $0.2-0.5 \% .^{10}$ They are typically self-limiting and do not require prolonged anticonvulsant treatment.

In our patient, the hyperdensity noted in the CT done soon after the seizure was probably due to leakage of contrast agent through the disrupted BBB. It does not indicate a cerebral infarction or haemorrhage. The patient's relatively intact neurological status and the resolution of the abnormalities on CT within 24-48 hours confirm our conclusions. The unilateral CT changes make one wonder if the ictal activity originated from the left hemisphere. However, an EEG performed 32-48 hours after the ictus failed to reveal any left hemispheric seizure focus. It is arguable that the EEG might have shown an abnormality if the study had been done soon after the ictus.

It is important to be aware of this possibility when interpreting abnormal CT scans performed soon after seizures. Failure to recognise this may lead to erroneous diagnosis and unwarranted expensive investigations. Proper diagnosis is not only cost-effective but will also avoid unnecessary distress both for the patient and the physician.

\section{Final diagnosis}

Anteroseptal myocardial infarction, contrast medium-induced seizure, and false-positive CT abnormalities due to post-seizure disruption of the blood-brain barrier.

Keywords: seizure; myocardial infarction; blood-brain barrier; contrast media

7 Sage MR, Drayer BP, Dubois RJ, et al. Increased permeability of brain blood barrier after carotid Renografin 76. Am $\mathcal{F}$ Neuroradiol 1981;2:272-4.

8 Scott WR. Seizures: a reaction to contrast media for computed tomography of the brain. Radiology 1980;137: 359 .

9 Sharp S, Stone J, Beach R. Contrast agent neurotoxicity presenting as subarachnoid hemorrhage. Neurology 1999;52: 1503-5.

10 Nelson M, Bartlett RJV, Lamb JT. Seizures after intravenous contrast media for cranial computed tomography. $\mathcal{F}$ Neurol Neurosurg Psychiatry 1989;52:1170-5. 


\section{Growth failure: an unusual clinical problem}

Shailendra Kumar Singh, Santosh Kumar Singh, Richa Chaturvedi, Manoj Chaudhari, M Rai, S K Singh, J K Agrawal

A 9-year-old boy presented with short stature and progressive genu valgum (figure 1). He had frontal bossing, rachitic rosary and widening of wrist. X-Rays of the wrist (figure 2) and knee joint (figure 3) showed cupping, fraying and widening of metaphyses. Laboratory investigations showed serum calcium $9.4 \mathrm{mg} / \mathrm{dl}$, phosphorus $2.2 \mathrm{mg} / \mathrm{dl}$, chloride $105 \mathrm{mmol} / \mathrm{l}$, potassium 3.0 $\mathrm{mmol} / \mathrm{l}$, alkaline phosphatase $1004 \mathrm{IU} / 1$, and albumin $4.1 \mathrm{~g} / \mathrm{dl} .24-\mathrm{Hour}$ urinary calcium excretion was $302 \mathrm{mg}$. Intact parathyroid hormone (iPTH) level was $50 \mathrm{pg} / \mathrm{ml}$. Blood pH was 7.32 and urine $\mathrm{pH}$ 8.0. Intravenous pyelogram was normal. A longitudinal ultrasound scan of the kidneys is shown in figure 4 . A test was done to confirm the diagnosis.

Institute of Medical Sciences, BHU,

Varanasi, India 221005

Department of

Endocrinology and

Human Metabolism

S K Singh

S K Singh

R Chaturvedi

S K Singh

J K Agrawal

Department of

Radiology

M Chaudhari

Department of

Medicine

M Rai

Correspondence to Prof J K Agrawal

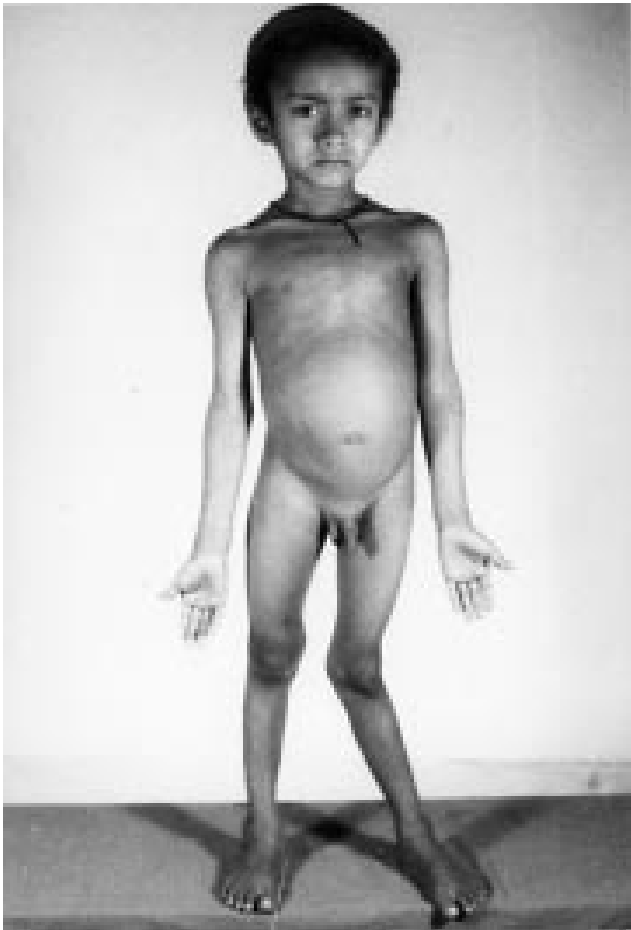

Figure 1 The patient (reproduced with his father's permission)

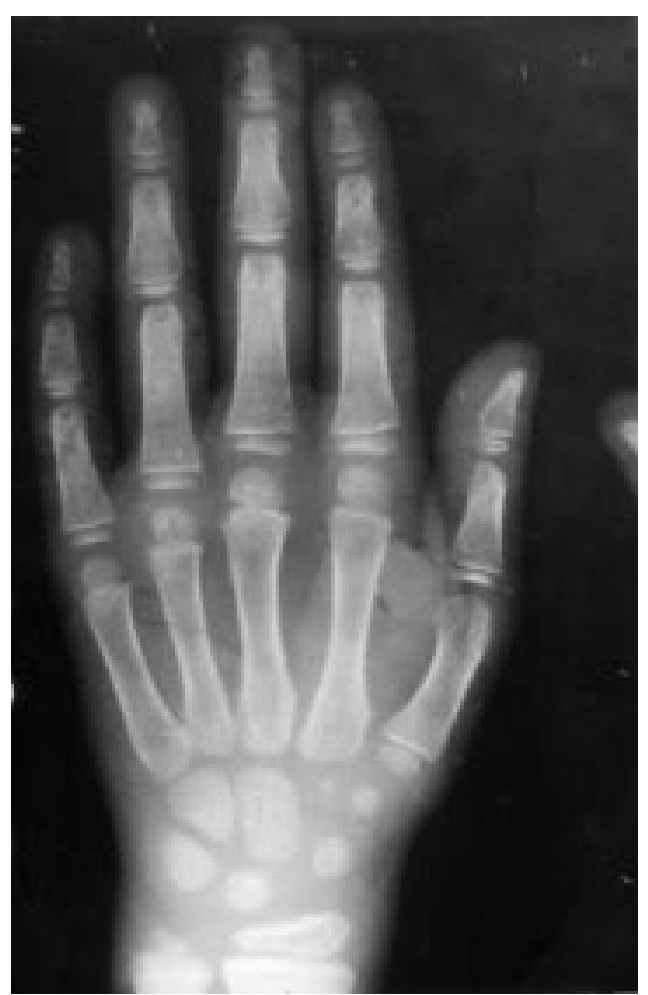

Figure 2 X-Ray of the wrist 


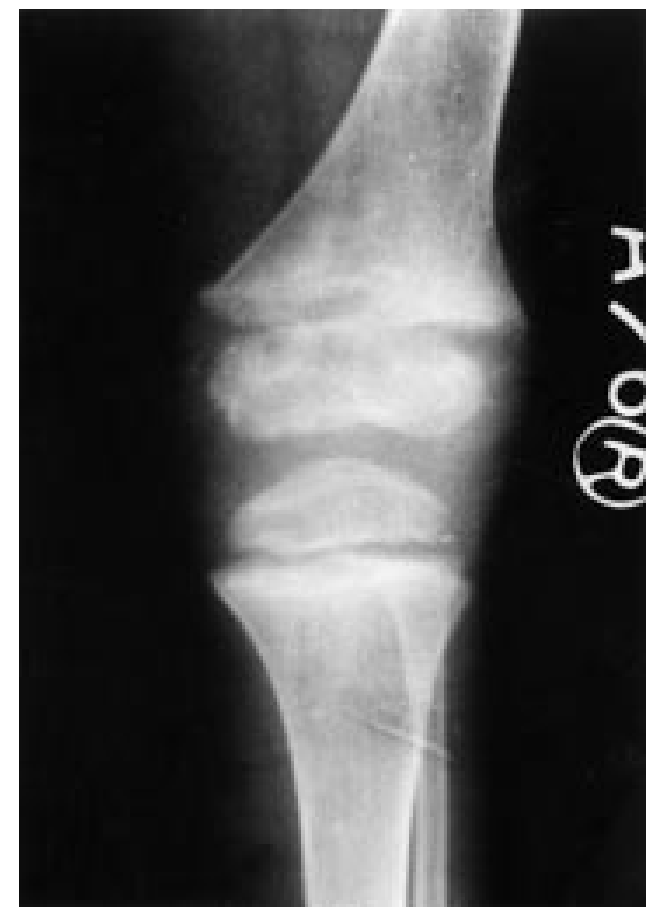

Figure 3 X-Ray of the knee joint

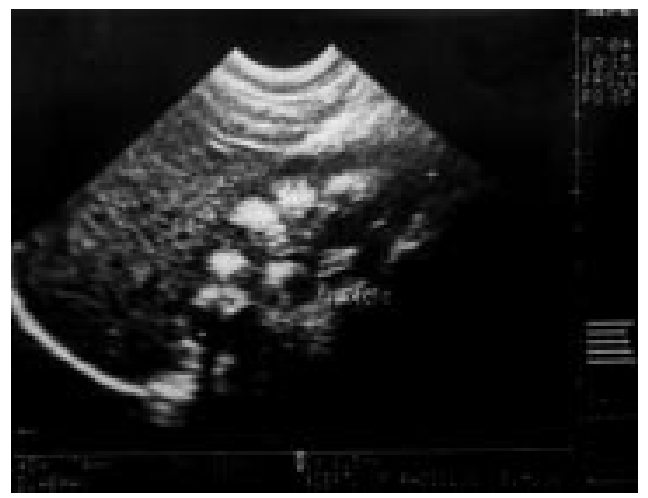

Figure 4 Longitudinal ultrasound scan of kidney

\section{Questions}

1 What does the abdominal ultrasound show? 2 What is the most likely diagnosis?

3 Name the test for confirmation of diagnosis. 4 How should this patient be managed? 
Answers

QUESTION 1

The ultrasound scan of the kidneys shows hyperechoic renal pyramids with normal echotexture of the cortex. Renal size on both sides appear normal with normal central sinus echos. Pelvicalyceal system of both kidneys are normal. These features are suggestive of medullary nephrocalcinosis.

QUESTION 2

The diagnosis is distal renal tubular acidosis (RTA) with rickets. The patient had normal iPTH and hyperchloraemic acidosis with nephrocalcinosis in the presence of clinical and biochemical changes of rickets.

QUESTION 3

The confirmatory test is the $\mathrm{NH}_{4} \mathrm{Cl}$ challenge test: $0.1 \mathrm{~g}(1.9 \mathrm{mmol}) \mathrm{NH}_{4} \mathrm{Cl} / \mathrm{kg}$ body weight is administered orally and blood and urine $\mathrm{pH}$ are followed for up to $6 \mathrm{~h}$. In distal RTA the urine $\mathrm{pH}$ remains higher than 5.5.

QUESTION 4

The disease can be treated with sodium bicarbonate or Shohl's solution $\mathrm{Na}^{+}$and $\mathrm{K}^{+}$citrate solution). The dose should be $0.5-2.0$ $\mathrm{mmol} / \mathrm{kg}$ body weight in four to five divided doses daily. The dose should be raised until acidosis and hypercalciuria are controlled. The patient should be followed by measurements of serum chloride, $\mathrm{pCO}_{2}$ in blood, and urinary calcium excretion, approximately twice yearly. Potassium supplementation is normally not required. The requirement of alkali usually rises during intercurrent illnesses. Corrective osteotomy may be done only when the rachitic changes have healed.

\section{Discussion}

RTA is a disorder of renal tubules in which the renal excretion of acid is reduced out of proportion to reduction of glomerular filtration rate. ${ }^{1}$ Metabolic acidosis results but, in contrast to renal failure, the anions that accompany surplus hydrogen ions in the blood such as sulphate and phosphate, are excreted normally and are unavailable to balance the fall in serum bicarbonate. Therefore the kidneys reabsorb chloride in unusually large amounts and serum chloride rises to preserve electroneutrality in the extracellular fluid. The result is hyperchloraemic acidosis, and unmeasured anion gap is normal. ${ }^{12}$ There are at least four types of RTA.

1 Coe FL Kathpalia S. Hereditary tubular disorders. In: Isselbocher KJ, Braunwald E, Wilson JD, Martin JB, Fanci AS, Kasper DL, eds. Harrison's Principles of internal me
13th edn, vol 2. McGraw-Hill Inc, 1994; pp 1323-9.

13th edn, vol 2. McGraw-Hill Inc, 1994; pp 1323-9.
2 Morris RCJr, Ives HE. Inherited disorders of the renal tubules. In: Brenner BM, Rector FC Jr, eds. The kidney, 5th edn. Philadelphia: WB Saunders, 1996; pp 1779-805.

3 DuBose TD Jr, Cogan MG, Rector FC Jr. Acid base disorders. In: Brenner BM, Rector FC Jr, eds. The kidney, 5th edn. Philadelphia: WB Saunders, 1996; p 958.

\section{Summary points}

- distal RTA is a rare condition

- in cases of rickets, if there is nephrocalcinosis in association with hyperchloraemic acidosis and alkaline urine, one should always suspect distal RTA

- the diagnosis is proved by a positive $\mathrm{NH}_{4} \mathrm{Cl}$ challenge test

- sodium bicarbonate or Shohl's solution is the cornerstone of treatment

Distal RTA is characterised by hypokalaemic hyperchloraemic metabolic acidosis and is due to selective deficiency in $\mathrm{H}^{+}$ion secretion in the distal nephrons. Despite acidosis, urinary $\mathrm{pH}$ is high and it cannot be acidified below $5.5{ }^{3}$ Urinary excretion of $\mathrm{NH}_{4}^{+}$is decreased and the urinary anion gap is positive. Chronic acidosis lowers tubular reabsorption of calcium, causing renal hypercalciuria. ${ }^{4}$ The hypercalciuria, alkaline urine and low level of urinary citrate cause calcium phosphate stones and nephrocalcinosis. ${ }^{5}$ Growth in children is stunted because of rickets. The bone disease is due to the acidosis-induced loss of bone mineral and inadequate production of $1,25(\mathrm{OH})_{2} \mathrm{D}_{3}{ }^{1}$ Since kidneys do not conserve potassium or concentrate the urine normally, polyuria and hypokalaemia occurs. The diagnosis is suggested by rickets or osteomalacia, hyperchloraemic acidosis, alkaline urine and calcium phosphate stones or nephrocalcinosis. $\mathrm{NH}_{4} \mathrm{Cl}$ challenge (see above) confirms the diagnosis. Although systemic acidosis worsens, urine $\mathrm{pH}$ does not fall below 5.5.

Distal RTA is treated with sodium bicarbonate and/or Shohl's solution $\left(\mathrm{Na}^{+}\right.$and $\mathrm{K}^{+}$ citrate), as stated above. The total dose of alkali should be raised until acidosis and hypercalciuria are both eliminated and patients should be followed by measurement of serum chloride and $\mathrm{pCO}_{2}$ in blood and urine calcium excretion approximately twice yearly. $\mathrm{K}^{+}$supplementation is required only when there is hypokalaemia-mediated muscle weakness and respiratory depression.

\section{Final diagnosis}

Rickets with distal renal tubular acidosis.

Keywords: renal tubular acidosis; nephrocalcinosis; ammonium chloride challenge test; rickets

4 Lemann J Jr, Litzow JR, Lennon EJ. The effects of chronic acid loads in normal man: further evidence for participation of bone minerals in the defense against chronic metabolic acidosis. J Clin Invest, 1966;45:1608-14.

5 Dedmon RE, Wrong O. The excretion of organic anion in renal tubular acidosis with particular reference to citrate. Clin Sci 1962;22:19-32. 


\title{
Mediastinal fatty tumour
}

\author{
John D Urschel, Dorothy M Urschel
}

A 60-year-old man complained of dyspnoea and recurrent pulmonary infections. He was an ex-smoker. Medical history was unremarkable. He denied cough, haemoptysis, and chest pain. Physical examination did not show any abnormalities. Basic laboratory studies and an electrocardiogram were normal. A chest X-ray showed a subtle mediastinal abnormality (figure 1), so a computed tomography (CT) scan of the chest was done. It showed a large mass in the mediastinum (figure 2).

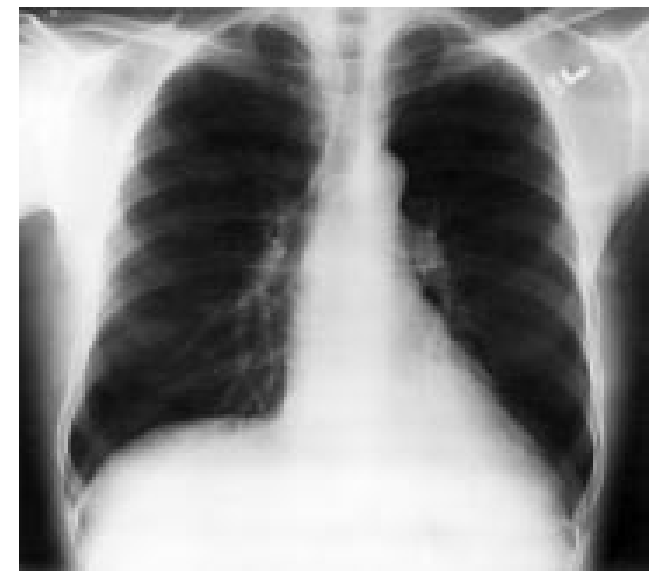

Figure 1 Chest X-ray shows a subtle abnormality overlying the left heart border

Correspondence to

JD Urschel, MD,

Department of Surgery, St

Joseph's Hospital, 50

Charlton Ave East,

Hamilton, Ontario, Canada

L8N 4A6

Accepted 17 May 1999

\section{Questions}

1 What is the differential diagnosis?

2 What biopsy techniques are appropriate?

3 What is the treatment?

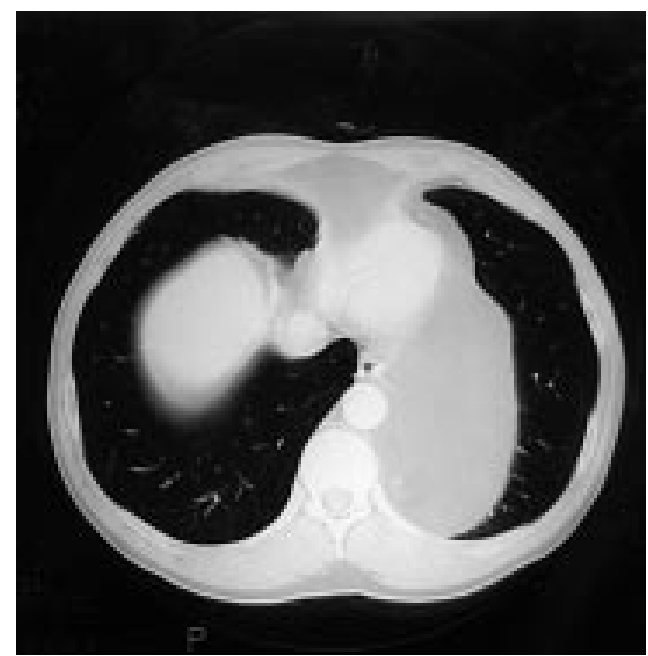

Figure 2 CT scan showing a large mediastinal mass 


\section{Answers}

QUESTION 1

The CT shows a homogenous mass with a tissue density consistent with fat. The differential diagnosis of mediastinal fatty tumours includes lipoma, liposarcoma, thymolipoma, and herniated peritoneal fat (hiatus or diaphragmatic hernia). Thymolipomas arise in the thymus gland (anterior mediastinum). Small fatty 'masses' in the inferior-posterior mediastinum often represent hiatus hernias, but it is unusual for large mediastinal fatty masses to be hernias. Large hernias usually consist of stomach or bowel, in addition to herniated peritoneal fat. The main differential diagnoses in this case are lipoma and liposarcoma.

QUESTION 2

Needle biopsies, either fine needle or core, are often inadequate for accurate diagnosis of lipomatous tumours. Low-grade liposarcomas are difficult to distinguish from benign lipomas on needle biopsy. Larger tissue specimens, from incisional or excisional biopsies, are usually required.

QUESTION 3

Complete excision (resection), when feasible, is the preferred treatment for lipomatous mediastinal tumours. This provides the pathologist with adequate tissue for diagnostic purposes, and the procedure is therapeutic as well.

\section{Discussion}

Mediastinal lipomas and liposarcomas are rare. ${ }^{1-3}$ They comprise less than $1 \%$ of all mediastinal tumours. A small tumour may be asymptomatic, but as the tumour increases in size compression of intrathoracic structures causes symptoms. Our patient's dyspnoea, for example, was relieved by tumour removal. Liposarcomas invade adjacent organs, so they are more likely to be symptomatic than benign lipomas, which simply compress adjacent structures.

Given the difficulty of accurate diagnosis with needle biopsies of lipomatous masses, complete excision is the diagnostic and therapeutic procedure of choice whenever possible. However, if the CT scan suggests that complete resection cannot be accomplished, there is little point in proceeding with a surgical procedure that leaves tumour in situ. ${ }^{2}$ In this situation an attempt at needle biopsy, or thoracoscopic incisional biopsy, are appropriate diagnostic procedures. Our patient's tumour was completely excised through a limited musclesparing thoracotomy. Final pathology showed a benign lipoma. The resection provided a diagnosis, relieved symptoms (dyspnoea) and prevented potential future problems from tumour growth.

Recurrence of a completely resected benign mediastinal lipoma is unlikely. However, if a liposarcoma had been found, the prognosis would have been quite poor. ${ }^{1}$ Mediastinal liposarcomas often invade adjacent vital organs, and microscopically complete tumour resection is difficult. Unfortunately, adjuvant treatment with radiation therapy or chemotherapy has not been very effective in intrathoracic mediastinal liposarcomas. ${ }^{14}$

\section{Final diagnosis}

Benign mediastinal lipoma.

Keywords: lipoma; liposarcoma; mediastinal tumours
3 Whooley BP, Urschel JD, Antkowiak JG, Anderson TM, Takita H. Primary tumors of the mediastinum. F Surg Oncol 1999;70:95-9.

Burt M, Ihde JK, Hajdu SI, et al. Primary sarcomas of the 1998;115:671-80.

2 Kato M, Saji S, Kunieda K, Yasue T, Nishio K, Adachi M Mediastinal lipoma: report of a case. Surg Today 1997;27: 766-8. 


\title{
Cavitary lung lesion in a patient with Sjögren's syndrome
}

\author{
J L Pérez-Castrillón, C Gonzalez-Castañeda, F Del Campo, J I Gonzalez, \\ J C Martín-Escudero, V Herreros
}

A 51-year-old woman was admitted to the hospital after 5 days of fever, cough, minor greenish sputum and right pleuritic pain. A non-smoker, she had a history of non-insulin-dependent diabetes and Sjögren's syndrome. Her temperature was $39^{\circ} \mathrm{C}$ and her blood pressure was 160/75 $\mathrm{mmHg}$. Pulmonary auscultation revealed right rales. The physical examination was otherwise unremarkable.

The white blood cell count was $12930 \times 10^{9} / 1$ (88\% neutrophils, $10 \%$ bands and $2 \%$ lymphocytes). The haemoglobin level was $8.8 \mathrm{~g} / \mathrm{dl}$ and the erythrocyte sedimentation rate was $118 \mathrm{~mm}$. The $\mathrm{PaO}_{2}$ when the patient was breathing ambient air was $63 \mathrm{mmHg}$. A Gram stain of the sputum revealed Gram-positive cocci. Three blood cultures obtained on admission yielded Streptococcus pneumoniae. The isolate was characterised as serotype 35. A chest X-ray taken on admission disclosed a parenchymal infiltrate in the right lower lobe (figure 1). A X-ray taken 14 days later displayed a cavity with an air crescent sign. A computed tomography (CT) scan showed lung tissue within the cavity (figure 2).

University Hospital

Rio Hortega,

University of

Valladolid, Valladolid,

Spain

Department of

Internal Medicine

J L Pérez-Castrillón

C Gonzalez-Castañeda

F Del Campo

J C Martín-Escudero

V Herreros

Department of

Radiology

J I Gonzalez

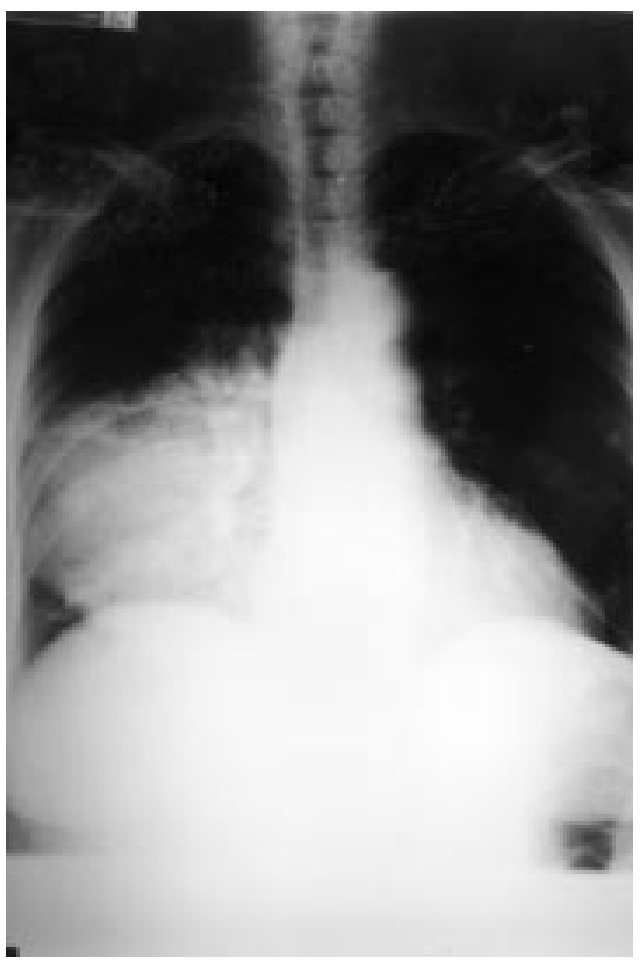

Figure 1 Admission chest X-ray

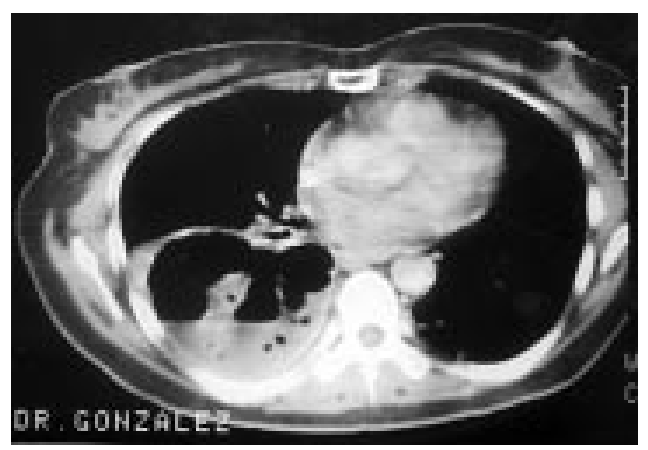

Figure 2 Thoracic CT scan

Correspondence to

José Luis Pérez Castrillón, University Hospital Rio

Hortega, Cardenal

Torquemeda s/n, 47014

Valladolid, Spain

Accepted 17 May 1999

\section{Questions}

1 What is the diagnosis?

2 What pulmonary alterations are associated with Sjögren's syndrome?

3 What is the management? 


\section{Answers}

QUESTION 1

The diagnosis is pneumococcal pneumonia complicated by pulmonary gangrene. Although a definitive diagnosis of pulmonary gangrene requires pathological confirmation, the radiologic findings are typical. The presence of a fluid-filled cavity in which irregular pieces of sloughed lung parenchyma float like icebergs is virtually diagnostic

QUESTION 2

The pulmonary abnormalities associated with Sjögren's syndrome are listed in the box.

\section{Pulmonary alterations associated with} Sjögren's syndrome

- xerotrachea

- chronic bronchitis

- atelectasis

- recurrent pneumonia

- interstitial alveolitis

- lymphoid interstitial pneumonia

- bronchiolitis obliterans with/without organising pneumonia

- interstitial fibrosis

- lymphoma

- amyloidosis

- pulmonary hypertension

QUESTION 3

Although successful medical management of pulmonary gangrene has been described, the majority of cases have been treated surgically, either by drainage or resection. Our patient was treated with antibiotic therapy (penicillin G 4 million $U$ six times daily for 4 weeks and tobramycin $80 \mathrm{mg}$ intravenously bid during the first 2 weeks) and percutaneous drainage.

\section{Discussion}

Pulmonary gangrene involves massive necrosis of lung parenchyma secondary to an overwhelming inflammatory pyogenic process that

1 Penner CH, Maycher B, Long R. Pulmonary gangrene. A complication of bacterial pneumonia. Chest 1994;105:56773

2 López-Contreras J, Ris J, Domingo P, Puig M, Martinez E. Tuberculous pulmonary gangrene. Report of a case and review. Clin Infect Dis 1994;18:243-5.

3 Yangco BG, Deresinski SC. Necrotizing or cavitating pneumonia due to Streptococcus pneumoniae: report of four cases and review of the literature. Medicine (Baltimore) 1980;59:449-57. is mediated by thrombosis of small and large arteries. The presence of lung tissue within a cavity is the result of secondary pulmonary infarction. The most frequently involved microorganisms are Klebsiella pneumoniae and Streptococcus pneumoniae. Other pathogens may be involved, including other Gram-negative bacilli, Mycobacterium tuberculosis and anaerobes. ${ }^{12} S$ pneumoniae has been described as the responsible microorganism in 11 cases, including the present one.

The pathophysiology of pulmonary gangrene is not well defined. The mechanism is multifactorial, including both microorganism and host factors. Serotype 3 pneumococci are known to cause more severe necrosis than other types. ${ }^{3}$ In our patient, not only were serotype 35 pneumococci isolated, moreover, she suffers from a primary Sjögren's syndrome. Pulmonary abnormalities in patients with primary Sjögren's syndrome have been well documented. ${ }^{4}$ Peripheral airways can be involved and the result is respiratory tract infection. Another factor associated with Sjögren's syndrome is the presence of pulmonary vasculitis . The vasculitis process, by means of local activation of the coagulation cascade, could explain the thrombosis and accompanying infarction. This is the first documented case of pulmonary gangrene associated with Sjögren's syndrome.

Although definitive diagnosis requires pathological confirmation, the typical roentgenographic picture is virtually diagnostic. ${ }^{2}$ The most useful procedure is CT, which shows the presence of lung tissue within the cavity. This cavity occurs in the upper lobes in $80 \%$ of cases.

The optimal therapeutic approach to infectious pulmonary gangrene has not been delineated accurately because of the extreme rarity of the condition. Antibiotic therapy alone may be effective, but in many cases urgent surgical measures are required. ${ }^{5}$ In this case, percutaneous drainage was a useful therapeutic procedure and no surgical measures were necessary.

\section{Final diagnosis}

Pulmonary gangrene.

Keywords: Sjögren's syndrome; pulmonary gangrene

4 Constantopoulos SH, Papadimitriou CS, Moutsopoulos HM. Respiratory manifestations in primary Sjögren's HM. Respiratory manifestations in primary sjogrene. A clinical, functional and histologic study. Chest syndrome. A clin

5 Refaely Y, Weissberg D. Gangrene of the lung: treatment in two stages. Ann Thorac Surg 1997;64:970-3. 


\title{
A case of painful thigh
}

\author{
Kuntal Chakravarty, S Gaddemsetty, S Shami
}

A 29-year-old previously fit Asian man presented with a 3-month history of diffuse pain in the right thigh, with no obvious swelling or paraesthesia. He had no pyrexia, nocturnal sweats or any significant weight loss. He had no history of travel abroad nor had he any contact with tuberculosis. Physical examination revealed a thin man with a temperature of $37^{\circ} \mathrm{C}$ and no palpable lymphadenopathy. Systemic examinations were otherwise unremarkable. Examination of the right thigh did not reveal any tenderness or swelling but straight leg raising on the right was restricted to $40^{\circ}$ (active) due to pain. Straight leg raising on the left was normal. There was no objective sensory impairment or abnormal reflexes in the lower limbs.

Initial investigations showed a haemoglobin of $11.8 \mathrm{~g} / \mathrm{dl}$ with a mean corpuscular volume of 62.3 (thallassaemic trait). His white cell and differential counts were normal. Plasma viscosity was raised at 2.2 (normal range up to 1.7). The following tests were normal or negative: rheumatoid factor, antinuclear factor, immunoglobulin electrophoresis and quantitative immunoglobulin assay, blood culture and creatine kinase; C-reactive protein was elevated at $61.9 \mathrm{mg} / 1$ (normal range 0-6). Radiographs of the chest, thoracic and lumbosacral spine and hip did not show any abnormality. An isotope bone scan (Tc99) was normal. Computed tomography (CT) of his lumbosacral spine failed to reveal any abnormality apart from a mild disc prolapse.

He was advised strong analgesics and physiotherapy and at review, 3 weeks later, an ill-defined painless fluctuant swelling on the right lateral thigh was discovered. A diagnostic aspiration revealed pus which was negative on Gram stain. Subsequent Zeil Nielson stain was also negative. An ultrasound scan of the right thigh showed extension of a cavity towards the right groin suggesting the possibility of a psoas abscess. A further CT scan of the abdomen (figures 1 and 2) failed to demonstrate any collection over the psoas or inside the pelvis and there was no connection with any intra-abdominal structure or the hip joint. However, the CT scan of the thigh muscles revealed a collection within the muscle planes of the glutei on the right extending to the right mid thigh (figure 3). A thoracic CT scan did not show any mediastinal, hilar or axillary lymphadenopathy. The lung fields were clear.

Seven hundred mls of pus was drained from the loculated swelling. There was no lymphadenopathy or intra-abdominal extension of the abscess cavity. The 'cavity' was loculated between the gluteus maximus and medius muscles.

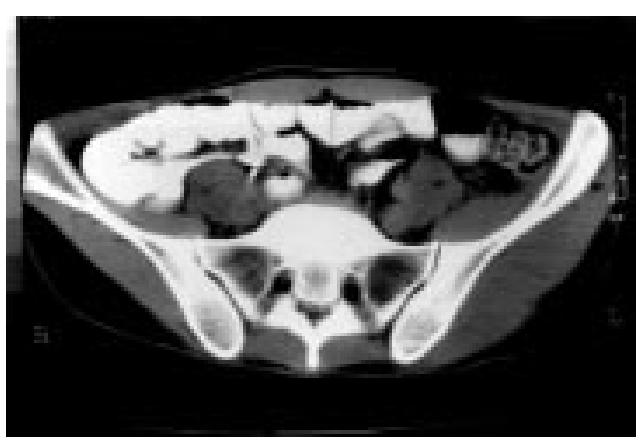

Figure 1 Abdominal CT scan

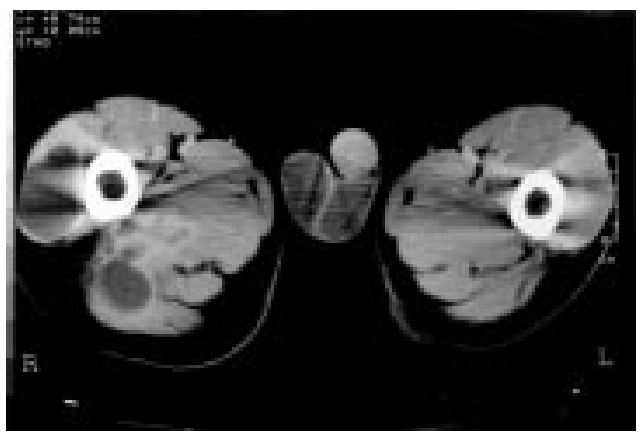

Figure 3 CT scan of the thigh muscles

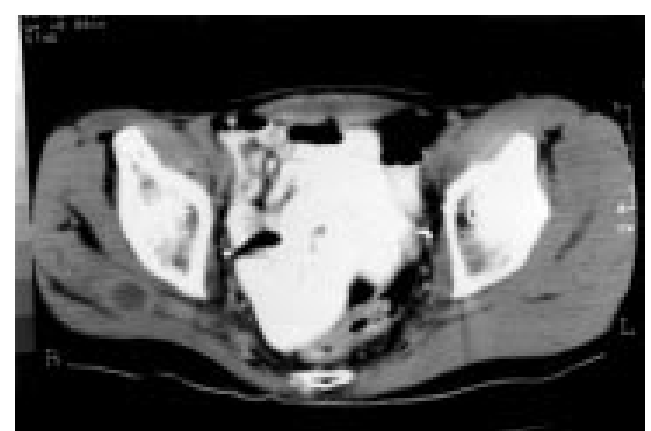

Figure 2 Abdominal CT scan

\section{Questions}

1 What do the CT scans in figures 1 and 2 show?

2 What is the most likely diagnosis on the basis of the CT scan findings? 


\section{Answers}

QUESTION 1

Figure 1 shows the normal outline of the psoas muscles with no evidence of intra-abdominal collection. Figure 2 shows a space-occupying lesion within the right gluteal region, which could either be an abscess cavity or a necrotic mass, not in communication with either a joint or intra-abdominal structure.

QUESTION 2

The most likely diagnosis is intramuscular abscess caused by a tubercular infection as the diagnostic aspiration yielded pus which as negative on Gram stain and also ordinary bacterial culture. The aspirated pus, however, showed profuse growth of acid fast bacilli, on culture. $\mathrm{He}$ was treated with rifampicin, ethambutol, isoniazid and pyrazinamide with complete resolution of his symptoms and no recurrence.

Primary intramuscular tubercular abscess is extremely rare and one should make a positive attempt to exclude intra-abdominal collection as psoas abscess may track down the groin or the thigh and present a similar clinical picture. It is not uncommon in patients who are immunosuppressed.

\section{Discussion}

Primary skeletal muscle tuberculosis is extremely rare and earlier studies have reported only four cases of muscle tuberculosis in 2224 autopsy specimens from tubercular patients and one in 60000 cases of all types of tuberculosis. ${ }^{12}$ By far the commonest site of involvement of the skeleton is the spine $(50 \%$ of cases), followed by hip and the knee. Our patient did not have any focal lesion on the spi-

1 Culotta A. La-tuberculosis muscuolare. Rev Pathol Tuberc 1929;3:1-26. nal CT scan and there was no evidence of any psoas abscess.

The involvement of skeletal muscle in tuberculosis is usually by a direct extension from a neighbouring joint or rarely by haematogenous spread. The pathophysiological mechanism is not clear but it is possible that high lactic acid content of muscles, absence of reticuloendothelial cells and lymphatic tissue in muscles associated with very rich blood supply may help towards the localisation of the bacteria in the muscles. Our patient was unusual as he had no systemic symptoms of any illness, no evidence of spinal tuberculosis, and no psoas abscess, which is one of the commonest presentations of swelling in the upper part of the thigh.

Discitis with or without psoas abscess is a common presenting feature in only $5-10 \%$ of cases of musculoskeletal tuberculosis. It is important to remember unusual presentations of tuberculosis in the appropriate racial background. It is important also to include symptomatic areas for scanning other than the typically described sites of spinal tuberculosis. Our patient was also somewhat unusual as he had no clinical or serological evidence of primary immune deficiency. He was neither a drug addict nor had any abnormal sexual behaviour. Although psoas abscess is a common cause of a swelling in the thigh, rare causes such as primary intramuscular tubercular abscess should also be considered in the differential diagnosis of painful swellings.

\section{Final diagnosis}

Intramuscular abscess caused by a tubercular infection.

Keywords: intramuscular abscess; tuberculosis

\footnotetext{
2 Petter CK. Some thoughts on tuberculosis of fascia and
} muscle. Lancet 1937;57:156-9. 


\title{
An unusual case of hypercalcaemia
}

\author{
R Gama, J Wright, G Ferns
}

A 53-year-old woman presented to her general practitioner with a 3-month history of lethargy. She was taking lithium carbonate ( $900 \mathrm{mg}$ daily for 9 years) and carbamezepine ( $400 \mathrm{mg}$ daily for 3 years) for manic depressive illness, and thyroxine (50 $\mu \mathrm{g}$ daily for 4 years) for primary hypothyroidism. She smoked 20 cigarettes a day. There was no abnormality on examination. Investigations revealed hypercalcaemia (serum calcium $3.05 \mathrm{mmol} / \mathrm{l}$ ). Subsequent relevant investigations are shown in the table. Chest, abdominal, hands and skull X-rays revealed no abnormality.

Table Biochemical and haematological investigations

\begin{tabular}{lll}
\hline Test & Result & Reference range \\
\hline Albumin $(\mathrm{g} / \mathrm{l})$ & 40 & $37-55$ \\
Corrected calcium $(\mathrm{mmol} / \mathrm{l})$ & 3.16 & $2.1-2.5$ \\
Uncorrected calcium $(\mathrm{mmol} / \mathrm{l})$ & 3.16 & $2.1-2.6$ \\
Phosphate $(\mathrm{mmol} / \mathrm{l})$ & 0.54 & $0.8-1.4$ \\
Intact parathyroid hormone (pmol/1) & 13.2 & $0.5-5.5$ \\
Magnesium $(\mathrm{mmol} / \mathrm{l})$ & 0.9 & $0.65-1.0$ \\
Creatinine $(\mu \mathrm{mol} / \mathrm{l})$ & 90 & $45-120$ \\
Alkaline phosphatase (IU/1) & 111 & $30-130$ \\
Protein electrophoresis & normal & $115-160$ \\
Haemoglobin $(\mathrm{g} / \mathrm{l})$ & 129 & $<5$ \\
ESR (mm) & 2 & $0.5-1.2$ \\
Lithium (mmol/l) & 0.7 & $60-160$ \\
Thyroxine (nmol/l) & 102 & $0.3-4.5$ \\
Thyroid stimulating hormone $(\mathrm{mU} / \mathrm{l})$ & 1.8 & $<20$ \\
Thyroid microsomal antibodies & negative & $<45$ \\
Bilirubin $(\mu$ mol/l) & $<20$ & $<55$ \\
Aspartate aminotransferase $(\mathrm{IU} / \mathrm{l})$ & 35 & \\
Gamma glutamyl transferase $(\mathrm{IU} / \mathrm{l})$ & 108 & \\
\hline
\end{tabular}

Department of Clinical

Biochemistry, Royal

Surrey County

Hospital, Guildford,

Surrey, UK

R Gama

J Wright

G Ferns

Correspondence to

Dr R Gama, Department of

Clinical Chemistry, New

Cross Hospital,

\section{Questions}

Wolverhampton, West

Midlands WV10 0QP, UK

Accepted 26 May 1999

1 What is the cause of the hypercalcaemia?

2 What further investigations would you request?

3 How would you manage the hypercalcaemia? 
Answers

QUESTION 1

An inappropriately elevated parathyroid hormone concentration in the presence of hypercalcaemia and normal renal function is diagnostic of primary hyperparathyroidism (HPT). Since the patient was on lithium, this raises the possibility of lithium-induced primary hyperparathyroidism.

QUESTION 2

Thallium-technetium subtraction scans and ultrasound scans are the more commonly used techniques to aid pre-operative localization of a possible adenoma. Computed axial tomographic scans and magnetic resonance imaging scans are also available, selective neck venous sampling for parathyroid hormone may be used and arteriography is rarely used. There is, however, some debate about the value of routine pre-operative localisation procedures in patients without previous neck surgery. In this case ultrasound of the neck and a thalliumtechnetium subtraction scan showed no abnormality.

QUESTION 3

Mild asymptomatic hypercalcaemia in elderly patients may just require monitoring of serum calcium. Indications for surgery include young patients, complications of HPT (bone disease, renal disease, urinary calculi, resistant hypertension, resistant peptic ulceration), symptomatic hypercalcaemia or persistent marked hypercalcaemia (serum $\mathrm{Ca}>3.00 \mathrm{mmol} / \mathrm{l}$ ). This patient was referred for surgical correction of her HPT because of her marked hypercalcaemia. Pre-operative evaluation confirmed HPT but it was noted that she was on lithium. Surgery was deferred and, after discussion with the psychiatrists, the lithium was discontinued and her manic-depressive illness managed with carbamezepine. This led to resolution of the hypercalcaemia within one month, which supported the diagnosis of lithium-induced HPT. We also considered the possibility that her primary hypothyroidism was lithium-induced but the patient refused to stop thyroxine therapy.

\section{Discussion}

Lithium is widely used in the management of acute mania, resistant depression, and prophylaxis of unipolar and bipolar affective disorders. Primary hyperparathyroidism is more prevalent in patients on lithium therapy. ${ }^{1}$ This

1 Taylor JW, Bell AJ. Lithium-induced parathyroid dysfunction: a case report and review of the literature. Ann Pharmation: a case report and

2 Nordenstrom J, Stigard K, Perbeck L, Willems J, BagedahlStrindlund $M$, Linder J. Hyperparathyroidism associated with treatment of manic-depressive disorders by lithium. Eur F Surg 1992;158:207-11.

3 Seely EW, Moore TJ, LeBoff MS, Brown EM. A single dose of lithium carbonate acutely elevates intact parathyroid hormone levels in men. Acta Endocrinol 1989;121:174-6.
Endocrine complications of lithium therapy ${ }^{5}$

thyroid disease: primary hypothyroidism (5-15\%)

- goitre $(5 \%)$

- thyrotoxicosis (rare)

- nephrogenic diabetes insipidus (20-25\%)

- hyperparathyroidism $(2.7 \%)$

- diabetes mellitus (rare)

increased prevalence may be due to unmasking of pre-existing parathyroid disease or to a direct effect of lithium on the parathyroid glands. Since hypercalcaemia can occur within a few days or several years of lithium therapy, it has been suggested that there are two varieties of lithium-induced HPT; an early onset HPT due to unmasking of an adenoma and a late-onset HPT due to parathyroid hyperplasia with possible end-point adenomatous change due to chronic lithium stimulation. ${ }^{2}$ Lithium may promote hypercalcaemia by two mechanisms. Lithium directly stimulates the secretion of parathyroid hormone, ${ }^{3}$ which may partly explain early-onset HPT. Lithium also decreases intracellular calcium uptake, thus reducing parathyroid sensitivity to circulating calcium levels leading to an increase in parathormone concentration. ${ }^{4}$ This resetting of the 'calciostat' may explain the pathogenesis of late-onset lithium-induced HPT by a mechanism similar to that found in other forms of HPT. Discontinuation of lithium usually corrects the HPT, but it need not be discontinued in mild asymptomatic uncomplicated hypercalcaemia provided serum calcium is monitored. ${ }^{1}$ The usual indications for surgery in HPT apply to lithium-induced HPT persisting after withdrawal of lithium or when it is medically inadvisable to stop lithium therapy. ${ }^{1}$

In summary, this case illustrates the importance of recognising lithium-induced HPT which may be reversible on stopping lithium and thus avoiding potentially unnecessary neck surgery.

\section{Final diagnosis}

Lithium-induced primary hyperparathyroidism.

Keywords: lithium; calcium; hyperparathyroidism

4 Mallette LE, Khouri K, Zengotita H, Hollis BW, Malani S Lithium increases intact and midregion parathyroid hormone and parathyroid volume. f Clin Endocrinol Metab 1989;68:654-60

5 Salata R, Klein I. Effect of lithium on the endocrine system; a review. F Lab Med 1987;110:130-6. 